\title{
A NEW FICTITIOUS DOMAIN APPROACH INSPIRED BY THE EXTENDED FINITE ELEMENT METHOD
}

\author{
JAROSLAV HASLINGER* AND YVES RENARD ${ }^{\dagger}$
}

\begin{abstract}
The purpose of this paper is to present a new fictitious domain approach inspired by the extended finite element method introduced by Moës, Dolbow and Belytschko in [18]. An optimal method is obtained thanks to an additional stabilization technique. Some a priori estimates are established and numerical experiments illustrate different aspects of the method. The presentation is made on a simple Poisson problem with mixed Neumann and Dirichlet boundary conditions. The extension to other problems or boundary conditions is quite straightforward.
\end{abstract}

Key words. fictitious domain, Xfem, approximation of elliptic problems, stabilization technique.

AMS subject classifications. $65 \mathrm{~N} 30,65 \mathrm{~N} 15$

1. Introduction. The extended finite element method (Xfem) was introduced by Moës, Dolbow and Belytschko in [18] and developed in many papers such as [5, $16,19,23,28]$. The first application of Xfem was done in structural mechanics when dealing with cracked domains. The specificity of the method is that it combines a levelset representation of the geometry of the crack (introduced in [25]) with an enrichment of a finite element space by singular and discontinuous functions. The enrichment of a finite element space with a singular function has been studied earlier by Strang and Fix in [26]. The originality of Xfem consists in a particular way of defining the enrichment via the multiplication by a partition of unity provided by basis functions of a Lagrange finite element method. Several strategies can be considered in order to extend or improve the original Xfem. Some of these strategies are presented in [16]. An a priori error estimate of a variant of Xfem for cracked domains is presented in [5].

In this work we adapt the techniques of Xfem to develop a new method allowing computations in domains whose boundaries are independent of the mesh. A similar attempt was done in $[17,27]$. Our goal is to develop a fully optimal method. It can be considered as a fictitious domain type method. Its advantage, compared to existing ones (see for instance $[11,13]$ ), is its ability to easily treat complex boundary conditions. The elementary matrices however have to be computed taking into account the geometry of the real boundary (in a nonlinear framework this disadvantage disappears since the tangent stiffness matrix has to be frequently re-computed).

Therefore, this method can be of interest for computational domains having moving boundaries or boundaries with a complex geometry and various conditions on them (Dirichlet, Neumann, Signorini, ...). In this paper, only Dirichlet and Neumann boundary conditions are considered. An extension to more complex boundary data is straightforward, at least from the implementation point of view.

The outline of this paper is as follows. In Section 1, we introduce the model problem which is represented by a simple Poisson equation with Neumann and Dirichlet boundary conditions. In Section 2 we describe the new method for a model problem without any stabilization. Section 3 is devoted to a convergence analysis of this approach. An abstract result is obtained which gives a convergence rate of order $\sqrt{h}$

\footnotetext{
${ }^{*}$ Department of Numerical Mathematics, Faculty of Mathematics and Physics, Sokolovská 83, 18675 Praha 8, Czech Republic (Jaroslav.Haslinger@mff.cuni.cz)

${ }^{\dagger}$ Université de Lyon, CNRS, INSA-Lyon, ICJ UMR5208, LaMCoS UMR5259, F-69621, Villeurbanne, France. (Yves.Renard@insa-lyon.fr)
} 
under reasonable regularity assumptions on the solution even for high order finite elements. The main part of this paper is Section 4 where a new stabilized method is introduced. Under appropriate assumptions we prove the stability of this formulation as well as optimal error estimates. In Section 5 we briefly mention details on the computational implementation. Numerical experiments for a model example with different choices of finite element spaces are presented in Section 6. The paper is completed with three appendices with proofs of trace theorems needed in the text.

2. Setting of the problem. We present a new approach for numerical realization of elliptic problems. The theoretical presentation is made for a two or threedimensional simply connected bounded domain $\Omega$ with a sufficiently smooth boundary. Let $\widetilde{\Omega} \subset \mathbb{R}^{d}(d=2$ or $d=3)$ be a rectangular or parallelepiped domain (the fictitious domain) containing $\Omega$ in its interior. We consider that the boundary $\Gamma$ of $\Omega$ is split into two parts $\Gamma_{N}$ and $\Gamma_{D}$ (see Fig. 2.1). It is assumed that $\Gamma_{D}$ has a nonzero $(d-1)$-dimensional Lebesgue measure.

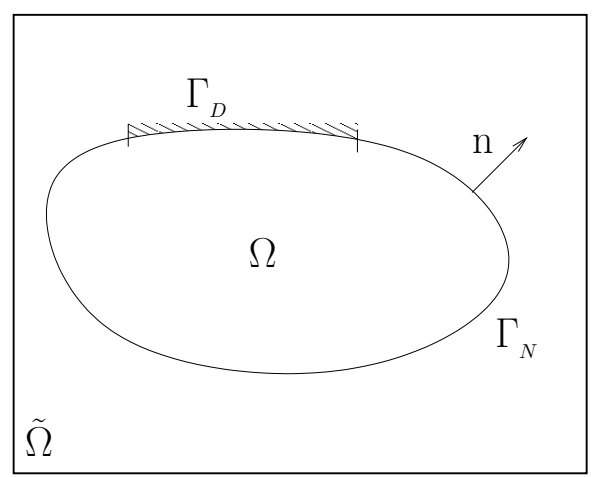

Figure 2.1. Fictitious and real domains.

Let us consider the following problem in $\Omega$ :

$$
\begin{aligned}
& \text { Find } u: \Omega \mapsto \mathbb{R} \text { such that } \\
& -\Delta u=f \quad \text { in } \Omega, \\
& u=0 \text { on } \Gamma_{D} \text {, } \\
& \partial_{n} u=g \text { on } \Gamma_{N} \text {, }
\end{aligned}
$$

where $f \in L^{2}(\Omega), g \in L^{2}\left(\Gamma_{N}\right)$ are given data and $n$ is the outward unit normal vector to $\Gamma$. The weak formulation of such a problem is well-known and reads as follows:

$$
\left\{\begin{array}{l}
\text { Find } u \in V_{0} \text { such that } \\
a(u, v)=l(v) \quad \forall v \in V_{0},
\end{array}\right.
$$

where

$$
\begin{gathered}
V=H^{1}(\Omega), \quad V_{0}=\left\{v \in V: v=0 \text { on } \Gamma_{D}\right\} \\
a(u, v)=\int_{\Omega} \nabla u . \nabla v d \Omega, \quad l(v)=\int_{\Omega} f v d \Omega+\int_{\Gamma_{N}} g v d \Gamma .
\end{gathered}
$$


It is also well-known that this problem can be expressed by means of the following mixed formulation:

$$
\left\{\begin{array}{l}
\text { Find } u \in V \text { and } \lambda \in W \text { such that } \\
a(u, v)+\langle\lambda, v\rangle_{W, X}=l(v) \forall v \in V, \\
\langle\mu, u\rangle_{W, X}=0 \quad \forall \mu \in W,
\end{array}\right.
$$

where $X=\left\{w \in L^{2}\left(\Gamma_{D}\right): \exists v \in V\right.$ such that $\left.w=v_{\Gamma_{D}}\right\}, W=X^{\prime}$ and $\langle\mu, v\rangle_{W, X}$ denotes the duality pairing between $W$ and $X$. Let

$$
V_{0}^{\#}=\left\{v \in V: \int_{\Gamma_{D}} v d \Gamma=0\right\} .
$$

Then $a(.,$.$) is coercive on V_{0}^{\#}$ (a direct consequence of Peetre-Tartar lemma, see [10] for instance), i.e. there exists $\alpha>0$ such that

$$
a(v, v) \geq \alpha\|v\|_{V}^{2} \quad \forall v \in V_{0}^{\#} .
$$

From this, the existence and uniqueness of a solution to Problem (2.5) follows. In addition, $\lambda=-\partial_{n} u$ on $\Gamma_{D}$. Problem (2.5) is also equivalent to the problem of finding a saddle point of the following Lagrangian on $V \times W$ :

$$
\mathcal{L}(v, \mu)=\frac{1}{2} a(v, v)+\langle\mu, v\rangle_{W, X}-l(v) .
$$

3. The new fictitious domain method. The new fictitious domain approach which will be studied in this paper requires the introduction of two finite dimensional finite element spaces $\widetilde{V}^{h} \subset H^{1}(\widetilde{\Omega})$ and $\widetilde{W}^{h} \subset L^{2}(\widetilde{\Omega})$ on the fictitious domain $\widetilde{\Omega}$. As $\widetilde{\Omega}$ can be a rectangular or parallelepiped domain, the ones can be defined on the same structured mesh $\mathcal{T}^{h}$ (see Fig. 3.1). Note that in the following, we only use the fact that the family of meshes is quasi-uniform (in the classical sense of Ciarlet $[6,7]$ ). Next we shall suppose that

$$
\widetilde{V}^{h}=\left\{v^{h} \in \mathcal{C}(\overline{\widetilde{\Omega}}):\left.v^{h}\right|_{T} \in P(T) \forall T \in \mathcal{T}^{h}\right\},
$$

where $P(T)$ is a finite dimensional space of regular functions such that $P(T) \supseteq P_{k}(T)$ for some $k \geq 1$, integer. The mesh parameter $h$ stands for $h=\max _{T \in \mathcal{T} h} h_{T}$ where $h_{T}$ is the diameter of $T$.

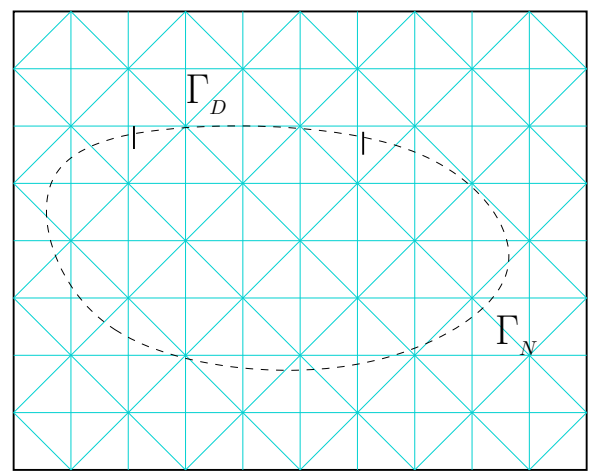

Figure 3.1. Example of a structured mesh. 
Then one can build

$$
V^{h}:=\widetilde{V}_{\left.\right|_{\Omega}}^{h}, \text { and } W^{h}:=\left.\widetilde{W}^{h}\right|_{\Gamma_{D}}
$$

which are natural discretizations of $V$ and $W$, respectively. An approximation of Problem (2.5) is defined as follows:

$$
\left\{\begin{array}{l}
\text { Find } u^{h} \in V^{h} \text { and } \lambda^{h} \in W^{h} \text { such that } \\
a\left(u^{h}, v^{h}\right)+\int_{\Gamma_{D}} \lambda^{h} v^{h} d \Gamma=l\left(v^{h}\right) \quad \forall v^{h} \in V^{h} \\
\int_{\Gamma_{D}} \mu^{h} u^{h} d \Gamma=0 \quad \forall \mu^{h} \in W^{h} .
\end{array}\right.
$$

Similarly to Xfem, where the shape functions of the finite element space is multiplied with an Heaviside function, this corresponds here to the multiplication of the shape functions with the characteristic function of $\Omega$.

4. Convergence analysis. Let us define the following space:

$$
V_{0}^{h}=\left\{v^{h} \in V^{h}: \int_{\Gamma_{D}} \mu^{h} v^{h} d \Gamma=0 \quad \forall \mu^{h} \in W^{h}\right\} .
$$

This space can be viewed to be a (nonconforming) discretization of $V_{0}$. In addition, we shall suppose that $\widetilde{W}^{h}$ and $\widetilde{V}^{h}$ are chosen in such a way that the following two conditions are satisfied for every $h>0$ :

$$
\begin{aligned}
& { }_{\left.\right|_{\Gamma_{D}} \in W^{h}} \\
& \bar{\mu}^{h} \in W^{h}: \int_{\Gamma_{D}} \bar{\mu}^{h} v^{h} d \Gamma=0 \quad \forall v^{h} \in V^{h} \Longrightarrow \bar{\mu}^{h}=0 .
\end{aligned}
$$

LEMma 4.1. The bilinear form $a(\cdot, \cdot)$ is uniformly $V_{0}^{h}$-elliptic, i.e. there exists $\alpha>0$ independent of $h$ such that

$$
a\left(v^{h}, v^{h}\right) \geq \alpha\left\|v^{h}\right\|_{V} \quad \forall v^{h} \in V_{0}^{h} .
$$

Proof. It follows from the fact that $V_{0}^{h} \subset V_{0}^{\#}$.

Proposition 1. Suppose that (4.2) and (4.3) are satisfied. Then the solution $\left(u^{h}, \lambda^{h}\right)$ to Problem (3.2) is unique and there exists a constant $C>0$ independent of $\widetilde{V}^{h}$ and $\widetilde{W}^{h}$ such that $\left({ }^{1}\right)$

$$
\left\|u^{h}\right\|_{V} \leq C\|l\|_{H^{-1}(\Omega)} .
$$

Proof. Since $1_{\left.\right|_{\Gamma_{D}}} \in W^{h}$, it follows from the last equality in (3.2) that $u^{h} \in V_{0}^{\#}$. The existence and uniqueness of $\left(u^{h}, \lambda^{h}\right)$ now follows from (4.3) and Lemma 4.1. The announced estimate comes from the fact that $a\left(u^{h}, u^{h}\right)=l\left(u^{h}\right)$.

We prove now the following abstract result (the extension of Cea's lemma).

\footnotetext{
${ }^{1}$ In what follows, the symbol $C$ will be used to denote a generic positive constant which does not depend on $h$ and which can take different values at different places of its appearance.
} 
Lemma 4.2. Let $(u, \lambda)$ and $\left(u^{h}, \lambda^{h}\right)$ be the solution to Problems (2.5) and (3.2), respectively. Suppose that (4.2) and (4.3) are satisfied. Then there exists a constant $C>0$ independent of $\widetilde{V}^{h}$ and $\widetilde{W}^{h}$ such that

$$
\left\|u-u^{h}\right\|_{V} \leq C\left(\inf _{v^{h} \in V_{0}^{h}}\left\|u-v^{h}\right\|_{V}+\sup _{v^{h} \in V_{0}^{h}, v^{h} \neq 0} \frac{\left|a\left(u, v^{h}\right)-l\left(v^{h}\right)\right|}{\left\|v^{h}\right\|_{V}}\right) .
$$

Proof. For a given function $v^{h} \in V_{0}^{h}$ one has:

$$
\begin{aligned}
\alpha\left\|u^{h}-v^{h}\right\|_{V}^{2} & \leq a\left(u^{h}-v^{h}, u^{h}-v^{h}\right) \\
& =a\left(u-v^{h}, u^{h}-v^{h}\right)+l\left(u^{h}-v^{h}\right)-a\left(u, u^{h}-v^{h}\right) .
\end{aligned}
$$

Thus

$$
\left\|u^{h}-v^{h}\right\|_{V} \leq C\left\|u-v^{h}\right\|_{V}+\sup _{w^{h} \in V_{0}^{h}, w^{h} \neq 0} \frac{\left|a\left(u, w^{h}\right)-l\left(w^{h}\right)\right|}{\left\|w^{h}\right\|_{V}} .
$$

From the triangle inequality $\left\|u-u^{h}\right\|_{V} \leq\left\|u-v^{h}\right\|_{V}+\left\|u^{h}-v^{h}\right\|_{V}$ we obtain the result.

REMARK 1. The term $\sup _{v^{h} \in V_{0}^{h}, v^{h} \neq 0} \frac{\left|a\left(u, v^{h}\right)-l\left(v^{h}\right)\right|}{\left\|v^{h}\right\|_{V}}$ is called a consistency error.

COROLlary 4.3. Under the assumptions of Lemma 4.2, there exists a constant $C>0$ independent of $\widetilde{V}^{h}$ and $\widetilde{W}^{h}$ such that

$$
\left\|u-u^{h}\right\|_{V} \leq C\left(\inf _{v^{h} \in V_{0}^{h}}\left\|u-v^{h}\right\|_{V}+\inf _{\mu^{h} \in W^{h}}\left\|\lambda-\mu^{h}\right\|_{W}\right) .
$$

Proof. Since $u$ is a solution to Problem (2.5) one has:

$$
a\left(u, v^{h}\right)=l\left(v^{h}\right)-\left\langle\lambda, v^{h}\right\rangle_{W, X} \quad \forall v^{h} \in V_{0}^{h} .
$$

The definition of $V_{0}^{h}$ yields:

$$
a\left(u, v^{h}\right)-l\left(v^{h}\right)=-\left\langle\lambda, v^{h}\right\rangle_{W, X}=\left\langle\mu^{h}-\lambda, v^{h}\right\rangle_{W, X} \quad \forall v^{h} \in V_{0}^{h} \quad \forall \mu^{h} \in W^{h},
$$

so that

$$
\left|a\left(u, v^{h}\right)-l\left(v^{h}\right)\right| \leq \inf _{\mu^{h} \in W^{h}}\left\|\lambda-\mu^{h}\right\|_{W}\left\|v^{h}\right\|_{V} \quad \forall v^{h} \in V_{0}^{h} .
$$

This, together with Lemma 4.2 gives (4.4).

We establish now the following convergence result.

Proposition 2. Suppose that (4.2) and (4.3) are satisfied and, in addition, let the system $\left\{V_{0}^{h}\right\},\left\{W^{h}\right\}, h \rightarrow 0+$ be dense in $V_{0}$ and $L^{2}\left(\Gamma_{D}\right)$, respectively. Then

$$
u^{h} \rightarrow u \quad \text { in } V, \quad h \rightarrow 0+,
$$

where $u$ and $u^{h}$ are the first components of the solution to (2.5) and (3.2), respectively. 
Proof. From Proposition 1 it follows that

$$
\left\|u^{h}\right\|_{V} \leq C \quad \forall h>0
$$

Thus there exists a subsequence, still denoted by the same symbol and an element $\bar{u} \in V$ such that

$$
u^{h} \rightarrow \bar{u} \quad \text { in } V, \quad h \rightarrow 0+.
$$

Since $\left\{W^{h}\right\}$ is dense in $L^{2}\left(\Gamma_{D}\right)$, for any $\mu \in L^{2}\left(\Gamma_{D}\right)$ there exists a sequence $\left\{\mu^{h}\right\}$, $\mu^{h} \in W^{h}$ such that

$$
\mu^{h} \rightarrow \mu \quad \text { in } L^{2}\left(\Gamma_{D}\right), \quad h \rightarrow 0+.
$$

Passing to the limit in the last equality in (3.2), using (4.5) and (4.6) we see that

$$
\int_{\Gamma_{D}} \mu \bar{u} d \Gamma=0 \quad \forall \mu \in L^{2}\left(\Gamma_{D}\right),
$$

which is equivalent to $\bar{u} \in V_{0}$. Let $\bar{v} \in V_{0}$ be given. Then, by the assumption there exists a sequence $\left\{\bar{v}^{h}\right\}, \bar{v}^{h} \in V_{0}^{h}$ such that

$$
\bar{v}^{h} \rightarrow \bar{v} \quad \text { in } V, \quad h \rightarrow 0+.
$$

Since $u^{h}$ solves $(3.2)$ we have

$$
a\left(u^{h}, \bar{v}^{h}\right)=l\left(\bar{v}^{h}\right) .
$$

From this, (4.5) and (4.7) we see that

$$
a(\bar{u}, \bar{v})=l(\bar{v}) \quad \forall \bar{v} \in V_{0},
$$

i.e. $u:=\bar{u}$ solves the original problem. As $u$ is unique, the whole sequence $\left\{u^{h}\right\}$ tends weakly to $u$ in $V$. Strong convergence of $\left\{u^{h}\right\}$ to $u$ follows from the fact that

$$
\left|u^{h}\right|_{1, \Omega} \rightarrow|u|_{1, \Omega}
$$

which is easy to verify.

In what follows, we shall estimate the first term on the right of (4.4). To simplify our presentation we shall consider a purely homogeneous Dirichlet problem, i.e. with $\Gamma_{D}=\Gamma$ and such that its solution $u$ belongs to $H^{1+d / 2+\varepsilon}(\Omega) \cap H_{0}^{1}(\Omega)$ for some $\varepsilon>0$ $\left(\Omega \subset \mathbb{R}^{d}\right)$. From the embedding theorem it immediately follows that

$$
u \in \mathcal{C}^{1}(\bar{\Omega})
$$

For $\delta>0$ given, we denote by $\Omega_{\delta}$ the subset of $\Omega$ :

$$
\Omega_{\delta}=\{x \in \Omega: \operatorname{dist}(x, \Gamma)>\delta\} .
$$

Let $\eta_{h}$ be a sufficiently smooth cut-off function:

$$
\eta_{h}=\left\{\begin{array}{l}
1 \text { in } \Omega \backslash \Omega_{2 h}, \\
0 \text { in } \Omega_{3 h} .
\end{array}\right.
$$


In $\Omega_{2 h} \backslash \Omega_{3 h}$ the function $\eta_{h}$ is defined in such a way that

$$
\left\|\nabla^{j} \eta_{h}\right\|_{\mathcal{C}(\bar{\Omega})} \leq \frac{C}{h^{j}}, \quad j=1,2 .
$$

The solution $u$ can be split and written in the form

$$
u=\eta_{h} u+\left(1-\eta_{h}\right) u \text {. }
$$

Next we show that

$$
\left\|\eta_{h} u\right\|_{V} \leq C \sqrt{h}, \quad h \rightarrow 0+.
$$

Indeed,

$$
\left\|\eta_{h} u\right\|_{V}^{2}=\|u\|_{1, \Omega \backslash \Omega_{2 h}}^{2}+\left\|\eta_{h} u\right\|_{1, \Omega_{2 h} \backslash \Omega_{3 h}}^{2} .
$$

From (4.8) it immediately follows that

$$
\|u\|_{1, \Omega \backslash \Omega_{2 h}}^{2} \leq C h, \quad h \rightarrow 0+.
$$

To get the estimate of the second term on the right of (4.11) it is sufficient to estimate the respective seminorm. It holds:

$$
\begin{aligned}
\left|\eta_{h} u\right|_{1, \Omega_{2 h} \backslash \Omega_{3 h}}^{2} & \leq C\left(\int_{\Omega_{2 h} \backslash \Omega_{3 h}}\left|\nabla \eta_{h}\right|^{2} u^{2} d \Omega+\int_{\Omega_{2 h} \backslash \Omega_{3 h}} \eta_{h}^{2}|\nabla u|^{2} d \Omega\right) \\
& \leq C h, \quad h \rightarrow 0+
\end{aligned}
$$

making use of (4.9) and the elementary estimate

$$
\max _{x \in \Omega_{2 h} \backslash \Omega_{3 h}}|u(x)| \leq C h,
$$

which holds in view of the fact that $u=0$ on $\Gamma$. From (4.12) and (4.13) we obtain (4.10).

Let $V_{00}^{h}$ be a subset of $V_{h}$ containing functions vanishing in a vicinity of $\Gamma$. More precisely,

$$
V_{00}^{h}=\left\{v^{h} \in V^{h}: v^{h}(a)=0 \quad \forall a \in \mathcal{N}^{h}\right\}
$$

where $\mathcal{N}^{h}$ is the set of those nodes of $\mathcal{T}^{h}$ which lie in $\Omega \backslash \Omega_{3 h / 2}$. Observe that $V_{00}^{h} \subset V_{0}^{h}$.

By $\Pi_{T} v$ we denote the standard $P$-Lagrange interpolate of $v$ on an element $T \in$ $\mathcal{T}^{h}, T \subset \Omega$. Since $P \supseteq P_{k}(k \geq 1)$ we know that

$$
\left\|v-\Pi_{T} v\right\|_{1, T} \leq C h_{T}\|v\|_{2, T},
$$

holds for any $v \in H^{2}(T), T \in \mathcal{T}^{h}$ and $T \subset \Omega$.

Proposition 3. Suppose that $\widetilde{V}^{h}$ is defined by (3.1), let (4.2) and (4.3) be satisfied and, in addition

$$
\inf _{\mu^{h} \in W^{h}}\left\|\lambda-\mu^{h}\right\|_{W} \leq C h^{\beta}, \quad \text { for some } \beta \geq 1 / 2 .
$$


Let the solution $u$ of (2.4) with $\Gamma=\Gamma_{D}$ be such that $u \in H^{1+d / 2+\varepsilon}(\Omega) \cap H_{0}^{1}(\Omega), \varepsilon>0$. Then

$$
\left\|u-u^{h}\right\|_{V} \leq C \sqrt{h}, \quad h \rightarrow 0+
$$

Proof. It is sufficient to estimate the first term on the right of (4.4). It holds:

$$
\begin{aligned}
\inf _{v^{h} \in V_{0}^{h}}\left\|u-v^{h}\right\|_{V} & \leq \inf _{v^{h} \in V_{00}^{h}}\left\|u-v^{h}\right\|_{V}=\inf _{v^{h} \in V_{00}^{h}}\left\|\eta_{h} u+\left(1-\eta_{h}\right) u-v^{h}\right\|_{V} \\
& \leq\left\|\eta_{h} u\right\|_{V}+\left\|\left(1-\eta_{h}\right) u-v^{h}\right\|_{V} \quad \forall v^{h} \in V_{00}^{h} .
\end{aligned}
$$

We construct $v^{h}$ as follows:

$$
\left.v^{h}\right|_{T}=\Pi_{T}\left(\left(1-\eta_{h}\right) u_{T}\right) \text { if } T \subset \Omega,
$$

otherwise we set $v^{h}=0$. It is readily seen that $v^{h} \in V_{00}^{h}$ and from (4.14) it follows that

$$
\left\|\left(1-\eta_{h}\right) u-v^{h}\right\|_{V} \leq C h\left\|\left(1-\eta_{h}\right) u\right\|_{2, \Omega} \leq C h\|u\|_{2, \Omega}+C h\left\|\eta_{h} u\right\|_{2, \Omega} .
$$

A direct computation shows that

$$
\left\|\eta_{h} u\right\|_{2, \Omega} \leq \frac{C}{\sqrt{h}}, \quad h \rightarrow 0+.
$$

Indeed, the $H^{2}(\Omega)$-seminorm can be estimated by

$$
\left|\eta_{h} u\right|_{2, \Omega}^{2} \leq C\left(\left\|\nabla^{2} \eta_{h}\right\|_{\mathcal{C}(\bar{\Omega})}^{2} \int_{\Omega_{2 h} \backslash \Omega_{3 h}} u^{2} d \Omega+\int_{\Omega_{2 h} \backslash \Omega_{3 h}}\left|\nabla \eta_{h}\right|^{2}|\nabla u|^{2} d \Omega+|u|_{2, \Omega}^{2}\right) \leq \frac{C}{h}
$$

as follows from (4.9) and (4.13). Using (4.17) in (4.16) we see that

$$
\left\|\left(1-\eta_{h}\right) u-v^{h}\right\|_{V} \leq C \sqrt{h}, \quad h \rightarrow 0+.
$$

From this and (4.10) we finally arrive at

$$
\inf _{v^{h} \in V_{0}^{h}}\left\|u-v^{h}\right\|_{V} \leq C \sqrt{h}
$$

The convergence rate given by the previous proposition is only of order $\sqrt{h}$. The numerical experiments of Section 7 show that this result, based on the classical formulation is optimal, in general. The aim of the next section is to propose a stabilization technique to overcome this limitation.

5. A stabilized formulation. In this section we adapt a stabilization technique presented by Barbosa and Hughes in $[2,3]$ in order to recover an optimal rate of convergence. Note that the link between this stabilization technique and the former Nitsche's method [20] has been established in [24]. Moreover it has been recently used to interface problems with nonmatching meshes in [4] and to elastostatic contact problems in [14]. We present its symmetric version although the nonsymmetric one can be considered in the same way. This technique is based on the addition of a 
supplementary term involving the normal derivative on $\Gamma_{D}$. In fact, we need a little bit more general definition. Let us suppose that we have at our disposal an operator

$$
R^{h}: V^{h} \longrightarrow L^{2}\left(\Gamma_{D}\right),
$$

which approximates the normal derivative on $\Gamma_{D}$ (i.e. for $v^{h} \in V^{h}$ converging to a sufficiently smooth function $v, R^{h}\left(v^{h}\right)$ tends to $\partial_{n} v$ in an appropriate sense). Several choices of $R^{h}$ will be proposed later. We suppose that the following estimate holds for this operator:

$$
h^{1 / 2}\left\|R^{h}\left(v^{h}\right)\right\|_{0, \Gamma_{D}} \leq C\left\|\nabla v^{h}\right\|_{0, \Omega} \quad \forall v^{h} \in V^{h}, \forall h>0 .
$$

To obtain the stabilized problem we replace the Lagrangian (2.7) by the following one

$$
\mathcal{L}_{h}\left(v^{h}, \mu^{h}\right)=\mathcal{L}\left(v^{h}, \mu^{h}\right)-\frac{\gamma}{2} \int_{\Gamma_{D}}\left(\mu^{h}+R^{h}\left(v^{h}\right)\right)^{2} d \Gamma, \quad v^{h} \in V^{h}, \mu^{h} \in W^{h},
$$

where for the sake of simplicity $\gamma:=h \gamma_{0}$ is chosen to be a positive constant over $\Omega$ (for non-uniform meshes, an element dependent parameter $\gamma=h_{T} \gamma_{0}$ is a better choice). The corresponding discrete problem reads as follows:

$$
\left\{\begin{array}{l}
\text { Find } u^{h} \in V^{h} \text { and } \lambda^{h} \in W^{h} \text { such that } \\
a\left(u^{h}, v^{h}\right)+\int_{\Gamma_{D}} \lambda^{h} v^{h} d \Gamma-\gamma \int_{\Gamma_{D}}\left(\lambda^{h}+R^{h}\left(u^{h}\right)\right) R^{h}\left(v^{h}\right) d \Gamma=l\left(v^{h}\right) \forall v^{h} \in V^{h}, \\
\int_{\Gamma_{D}} \mu^{h} u^{h} d \Gamma-\gamma \int_{\Gamma_{D}}\left(\lambda^{h}+R^{h}\left(u^{h}\right)\right) \mu^{h} d \Gamma=0 \quad \forall \mu^{h} \in W^{h} .
\end{array}\right.
$$

As in [2], let us define the form $\mathcal{B}_{h}:\left(V^{h} \times W^{h}\right)^{2} \longrightarrow \mathbb{R}$ by

$$
\begin{aligned}
\mathcal{B}_{h}\left(u^{h}, \lambda^{h} ; v^{h}, \mu^{h}\right):= & a\left(u^{h}, v^{h}\right)+\int_{\Gamma_{D}} \lambda^{h} v^{h} d \Gamma+\int_{\Gamma_{D}} \mu^{h} u^{h} d \Gamma \\
& -\gamma \int_{\Gamma_{D}}\left(\lambda^{h}+R^{h}\left(u^{h}\right)\right)\left(\mu^{h}+R^{h}\left(v^{h}\right)\right) d \Gamma .
\end{aligned}
$$

Then, (5.2) is equivalent to

$$
\left\{\begin{array}{l}
\text { Find } u^{h} \in V^{h} \text { and } \lambda^{h} \in W^{h} \text { such that } \\
\mathcal{B}_{h}\left(u^{h}, \lambda^{h} ; v^{h}, \mu^{h}\right)=l\left(v^{h}\right), \quad \forall\left(v^{h}, \mu^{h}\right) \in V^{h} \times W^{h} .
\end{array}\right.
$$

Moreover, this formulation is consistent in the sense that the solution $(u, \lambda)$ to problem (2.5) satisfies

$$
\overline{\mathcal{B}}_{h}\left(u, \lambda ; v^{h}, \mu^{h}\right)=l\left(v^{h}\right), \quad \forall v^{h} \in V^{h}, \forall \mu^{h} \in W^{h},
$$

provided that $\lambda \in L^{2}\left(\Gamma_{D}\right)$ with $\overline{\mathcal{B}}_{h}$ having the same definition as $\mathcal{B}_{h}$ but replacing $R^{h}(u)$ by $\partial_{n} u$.

The following hypothesis on the approximation property of $W^{h}$ will be needed to get an abstract result. Let $P^{h}: L^{2}\left(\Gamma_{D}\right) \longrightarrow W^{h}$ be the $L^{2}$-projection on $W^{h}$. We suppose that there exists a constant $C>0$ independent of $h$ such that

$$
\left\|P^{h} v-v\right\|_{0, \Gamma_{D}} \leq C h^{1 / 2}\|v\|_{1 / 2, \Gamma_{D}}, \quad \forall v \in H^{1 / 2}\left(\Gamma_{D}\right) .
$$


This allows to establish the following "inf-sup" property of $\mathcal{B}_{h}$.

Lemma 5.1. Let hypotheses (4.2), (5.1) and (5.5) be satisfied. Then for $\gamma_{0}>0$ sufficiently small there exists a constant $C>0$ independent of $h$ such that

$$
\sup _{(0,0) \neq\left(z^{h}, \eta^{h}\right) \in V^{h} \times W^{h}} \frac{\mathcal{B}_{h}\left(v^{h}, \mu^{h} ; z^{h}, \eta^{h}\right)}{\left|\left\|\left(z^{h}, \eta^{h}\right)\right\|\right|} \geq C\left|\left\|\left(v^{h}, \mu^{h}\right)\right\|\right|,
$$

where $\left|\left\|\left(z^{h}, \eta^{h}\right)\right\|\right|^{2}:=\left\|z^{h}\right\|_{V}^{2}+h^{-1}\left\|z^{h}\right\|_{0, \Gamma_{D}}^{2}+h\left\|\eta^{h}\right\|_{0, \Gamma_{D}}^{2}$.

Proof. The proof is an adaptation of the one in [24], Lemma 5. First of all, for $\left(v^{h}, \mu^{h}\right) \in V^{h} \times W^{h}$ arbitrary, $\gamma_{0}>0$ sufficiently small and from (5.1) one has

$$
\begin{aligned}
\mathcal{B}_{h}\left(v^{h}, \mu^{h} ; v^{h},-\mu^{h}\right) & =\left\|\nabla v^{h}\right\|_{0, \Omega}^{2}+\gamma_{0} h\left\|\mu^{h}\right\|_{0, \Gamma_{D}}^{2}-\gamma_{0} h\left\|R^{h}\left(v^{h}\right)\right\|_{0, \Gamma_{D}}^{2} \\
& \geq C\left(\left\|\nabla v^{h}\right\|_{0, \Omega}^{2}+h\left\|\mu^{h}\right\|_{0, \Gamma_{D}}^{2}\right) .
\end{aligned}
$$

Next, from (5.1) and the Young inequality we get for $\bar{\mu}^{h}:=h^{-1} P^{h} v^{h}$ :

$$
\begin{aligned}
\mathcal{B}_{h}\left(v^{h}, \mu^{h} ; 0, \bar{\mu}^{h}\right) & =\int_{\Gamma_{D}} \bar{\mu}^{h} v^{h} d \Gamma-\gamma \int_{\Gamma_{D}}\left(\mu^{h}+R^{h}\left(v^{h}\right)\right) \bar{\mu}^{h} d \Gamma \\
& \geq h^{-1}\left\|P^{h} v^{h}\right\|_{0, \Gamma_{D}}^{2}-C\left(\left\|\nabla v^{h}\right\|_{0, \Omega}+h^{1 / 2}\left\|\mu^{h}\right\|_{0, \Gamma_{D}}\right) h^{-1 / 2}\left\|P^{h} v^{h}\right\|_{0, \Gamma_{D}} \\
& \geq h^{-1}\left\|P^{h} v^{h}\right\|_{0, \Gamma_{D}}^{2}-\frac{C^{2}}{2}\left(\left\|\nabla v^{h}\right\|_{0, \Omega}+h^{1 / 2}\left\|\mu^{h}\right\|_{0, \Gamma_{D}}\right)^{2}-\frac{h^{-1}}{2}\left\|P^{h} v^{h}\right\|_{0, \Gamma_{D}}^{2} \\
& \geq \frac{h^{-1}}{2}\left\|P^{h} v^{h}\right\|_{0, \Gamma_{D}}^{2}-\bar{C}\left(\left\|\nabla v^{h}\right\|_{0, \Omega}^{2}+h\left\|\mu^{h}\right\|_{0, \Gamma_{D}}^{2}\right) .
\end{aligned}
$$

We now take $\left(z^{h}, \eta^{h}\right)=\left(v^{h},-\mu^{h}+\delta \bar{\mu}^{h}\right)$ in (5.6) with $\delta>0$. Using (5.7), (5.8) and $\delta$ sufficiently small one has:

$$
\begin{aligned}
\mathcal{B}_{h}\left(v^{h}, \mu^{h} ; z^{h}, \eta^{h}\right) & =\mathcal{B}_{h}\left(v^{h}, \mu^{h}, v^{h},-\mu^{h}\right)+\delta \mathcal{B}_{h}\left(v^{h}, \mu^{h}, 0, \bar{\mu}^{h}\right) \\
& \geq C\left(\left\|\nabla v^{h}\right\|_{0, \Omega}^{2}+h^{-1}\left\|P^{h} v^{h}\right\|_{0, \Gamma_{D}}^{2}+h\left\|\mu^{h}\right\|_{0, \Gamma_{D}}^{2}\right) .
\end{aligned}
$$

Since $\{1\} \subset W^{h}$ then for the $L^{2}$-projection of $v^{h}$ on $\{1\}$ we obtain:

$$
\left\|P^{h} v^{h}\right\|_{0, \Gamma_{D}}^{2} \geq \int_{\Gamma_{D}}\left(\frac{1}{\left|\Gamma_{D}\right|} \int_{\Gamma_{D}} v^{h} d \Gamma\right)^{2} d \Gamma=\frac{1}{\left|\Gamma_{D}\right|}\left(\int_{\Gamma_{D}} v^{h} d \Gamma\right)^{2} .
$$

Let $\beta>0$ be sufficiently small. Then it holds:

$$
\begin{aligned}
\left\|\nabla v^{h}\right\|_{0, \Omega}^{2}+h^{-1}\left\|P^{h} v^{h}\right\|_{0, \Gamma_{D}}^{2}= & \left\|\nabla v^{h}\right\|_{0, \Omega}^{2}+(1-\beta) h^{-1}\left\|P^{h} v^{h}\right\|_{0, \Gamma_{D}}^{2} \\
& +\beta h^{-1}\left\|P^{h} v^{h}-v^{h}+v^{h}\right\|_{0, \Gamma_{D}}^{2} \\
\geq & \left\|\nabla v^{h}\right\|_{0, \Omega}^{2}+(1-\beta) \frac{1}{\left|\Gamma_{D}\right| \operatorname{diam}(\Omega)}\left(\int_{\Gamma_{D}} v^{h} d \Gamma\right)^{2} \\
& +\beta h^{-1}\left(\left\|v^{h}\right\|_{0, \Gamma_{D}}^{2}-\left\|P^{h} v^{h}-v^{h}\right\|_{0, \Gamma_{D}}^{2}\right) \\
\geq & C\left(\left\|v^{h}\right\|_{V}^{2}+\beta h^{-1}\left(\left\|v^{h}\right\|_{0, \Gamma_{D}}^{2}-h\left\|v^{h}\right\|_{1 / 2, \Gamma_{D}}^{2}\right)\right) \\
\geq & C\left(\left\|v^{h}\right\|_{V}^{2}+h^{-1}\left\|v^{h}\right\|_{0, \Gamma_{D}}^{2}\right),
\end{aligned}
$$

where we used (5.5), the fact that $\left(\left\|\nabla v^{h}\right\|_{0, \Omega}^{2}+(1-\beta) \frac{1}{\left|\Gamma_{D}\right| \operatorname{diam}(\Omega)}\left(\int_{\Gamma_{D}} v^{h} d \Gamma\right)^{2}\right)^{1 / 2}$ is an equivalent norm on $V$ and the trace theorem. Finally, one obtains (5.6) combining (5.9) and (5.13) together with the fact that $\left|\left\|\left(z^{h}, \eta^{h}\right)\right\|\right| \leq C\left|\left\|\left(v^{h}, \mu^{h}\right)\right\|\right|$. 
REMARK 2. The inf-sup condition straightforwardly ensures the existence and uniqueness of a solution to the discrete problem (5.2) for $\gamma_{0}>0$ sufficiently small.

Now, we can prove the following abstract error estimate.

THEOREM 5.2. Let (4.2), (5.1) and (5.5) be satisfied and $\gamma_{0}>0$ be sufficiently small. If $(u, \lambda)$ is the solution to Problem (2.5) such that $\lambda \in L^{2}\left(\Gamma_{D}\right)$ then there exists a constant $C>0$ independent of $h$ and $(u, \lambda)$ such that the following estimate holds:

$\left|\left\|\left(u-u^{h}, \lambda-\lambda^{h}\right)\right\|\right| \leq C \inf _{v^{h} \in V^{h}, \mu^{h} \in W^{h}}\left(\left|\left\|\left(u-v^{h}, \lambda-\mu^{h}\right)\right\|\right|+h^{1 / 2}\left\|R^{h}\left(v^{h}\right)-\partial_{n} u\right\|_{0, \Gamma_{D}}\right)$.

Proof. From (5.4) it follows that

$$
\overline{\mathcal{B}}_{h}\left(u, \lambda, z^{h}, \eta^{h}\right)=\mathcal{B}_{h}\left(u^{h}, \lambda^{h}, z^{h}, \eta^{h}\right) \quad \forall\left(z^{h}, \eta^{h}\right) \in V^{h} \times W^{h} .
$$

Thus for any $\left(v^{h}, \mu^{h}\right) \in V^{h} \times W^{h}$ one has

$\mathcal{B}_{h}\left(v^{h}, \mu^{h}, z^{h}, \eta^{h}\right)-\overline{\mathcal{B}}_{h}\left(u, \lambda, z^{h}, \eta^{h}\right)=\mathcal{B}_{h}\left(v^{h}-u^{h}, \mu^{h}-\lambda^{h}, z^{h}, \eta^{h}\right) \quad \forall\left(z^{h}, \eta^{h}\right) \in V^{h} \times W^{h}$.

A direct computation leads to

$$
\begin{aligned}
\mathcal{B}_{h}\left(v^{h}, \mu^{h} ; z^{h}, \eta^{h}\right)-\overline{\mathcal{B}}_{h}\left(u, \lambda ; z^{h}, \eta^{h}\right) \leq & C\left(\left|\left\|\left(u-v^{h}, \lambda-\mu^{h}\right)\right\|\right|\right. \\
& \left.+h^{1 / 2}\left\|R^{h}\left(v^{h}\right)-\partial_{n} u\right\|_{0, \Gamma_{D}}\right)\left|\left\|\left(z^{h}, \eta^{h}\right)\right\|\right| .
\end{aligned}
$$

Further

$$
\begin{aligned}
\|\|\left(u-u^{h}, \lambda-\lambda^{h}\right) \| \mid \leq & \left|\left\|\left(u-v^{h}, \lambda-\mu^{h}\right)\right\|\right|+\left|\left\|\left(v^{h}-u^{h}, \mu^{h}-\lambda^{h}\right)\right\|\right| \\
\leq & \left|\left\|\left(u-v^{h}, \lambda-\mu^{h}\right)\right\|\right| \\
& \quad+C \sup _{(0,0) \neq\left(z^{h}, \eta^{h}\right) \in V^{h} \times W^{h}} \frac{\mathcal{B}_{h}\left(v^{h}-u^{h}, \mu^{h}-\lambda^{h} ; z^{h}, \eta^{h}\right)}{\left|\left\|\left(z^{h}, \eta^{h}\right)\right\|\right|} \\
& \leq C\left(\left|\left\|\left(u-v^{h}, \lambda-\mu^{h}\right)\right\|\right|+h^{1 / 2}\left\|R^{h}\left(v^{h}\right)-\partial_{n} u\right\|_{0, \Gamma_{D}}\right)
\end{aligned}
$$

holds for any $\left(v^{h}, \mu^{h}\right) \in V^{h} \times W^{h}$.

In the rest of this section we show how to use the abstract result of Theorem 5.2 to establish an optimal a priori error estimate for the following standard finite element spaces:

$$
\begin{gathered}
\widetilde{V}^{h}=\left\{v^{h} \in \mathcal{C}(\widetilde{\Omega}):\left.v^{h}\right|_{T} \in P_{k_{u}}(T) \forall T \in \mathcal{T}^{h}\right\}, \quad k_{u} \geq 1, \\
\widetilde{W}^{h}=\left\{\mu^{h} \in L^{2}(\widetilde{\Omega}):\left.\mu^{h}\right|_{T} \in P_{k_{\lambda}}(T) \forall T \in \mathcal{T}^{h}\right\}, \quad k_{\lambda} \geq 0 .
\end{gathered}
$$

In order to estimate the boundary terms, we shall need the following classical estimate which is satisfied for any $T \in \mathcal{T}^{h}$ and any $w \in H^{1}(T)$ provided that $\Gamma_{D}$ is smooth enough (see Appendix A for the proof):

$$
\|w\|_{0, \Gamma_{D} \cap T}^{2} \leq C\left(h_{T}^{-1}\|w\|_{0, T}^{2}+h_{T}\|w\|_{1, T}^{2}\right)
$$

Let $k=\min \left(k_{u}, k_{\lambda}+1\right)$ and consider two continuous extension operators:

$$
T_{u}^{k}: H^{k+1}(\Omega) \longrightarrow H^{k+1}(\widetilde{\Omega}),
$$




$$
T_{\lambda}^{k}: H^{k-1 / 2}\left(\Gamma_{D}\right) \longrightarrow H^{k}(\widetilde{\Omega}),
$$

where $H^{k-1 / 2}\left(\Gamma_{D}\right)$ stands for the space of traces on $\Gamma_{D}$ of functions from $H^{k}(\Omega)$. Due to Calderón's extension theorem, it is always possible to build such operators provided that the domain $\Omega$ has the uniform cone property (see [1] for instance). This allows us to define the following interpolation operators on $\widetilde{V}^{h}$ and $\widetilde{W}^{h}$ :

$$
\begin{gathered}
\widetilde{\Pi}_{u}^{k, h}(v):=\Pi^{k, h}\left(T_{u}^{k}(v)\right) \quad \forall v \in H^{k+1}(\Omega), \\
\widetilde{\Pi}_{\lambda}^{k, h}(\mu):=\Pi^{k-1, h}\left(T_{\lambda}^{k}(\mu)\right) \quad \forall \mu \in H^{k-1 / 2}\left(\Gamma_{D}\right),
\end{gathered}
$$

where $\Pi^{k, h}$ stands for the standard Lagrange interpolation operator by piecewise polynomial functions of degree less or equal $k$ defined on the mesh $\mathcal{T}^{h}$. An exception has to be done for $k=1$ when the Lagrange interpolation operator will be replaced by Clément's one for the interpolation of the multiplier since functions from $H^{1}(\widetilde{\Omega})$ are not generally continuous (see [8]). Due to the known approximation properties of these operators on regular families of meshes (see [7] and [8]), one has for any $v \in H^{k+1}(\Omega)$ :

$$
\begin{aligned}
\left\|\widetilde{\Pi}_{u}^{k, h}(v)-v\right\|_{V} & \leq\left\|\widetilde{\Pi}_{u}^{k, h}(v)-T_{u}^{k}(v)\right\|_{1, \widetilde{\Omega}} \\
& \leq C h^{k}\left\|T_{u}^{k}(v)\right\|_{k+1, \widetilde{\Omega}} \leq C h^{k}\|v\|_{k+1, \Omega}
\end{aligned}
$$

and for any $\mu \in H^{k-1 / 2}\left(\Gamma_{D}\right)$ taking into account (5.16):

$$
\begin{aligned}
\left\|\widetilde{\Pi}_{\lambda}^{k, h}(\mu)-\mu\right\|_{0, \Gamma_{D}}^{2} & \leq C \sum_{T \in \mathcal{T}^{h}}\left(h^{-1}\left\|\widetilde{\Pi}_{\lambda}^{k, h}(\mu)-T_{\lambda}^{k}(\mu)\right\|_{0, T}^{2}+h\left\|\widetilde{\Pi}_{\lambda}^{k, h}(\mu)-T_{\lambda}^{k}(\mu)\right\|_{1, T}^{2}\right) \\
& \leq C h^{2 k-1}\left\|T_{\lambda}^{k}(\mu)\right\|_{k, \widetilde{\Omega}}^{2} \leq C h^{2 k-1}\|\mu\|_{k-1 / 2, \Gamma_{D}}^{2} .
\end{aligned}
$$

In the same way one can derive the estimate $\left\|\widetilde{\Pi}_{u}^{k, h}(v)-v\right\|_{0, \Gamma_{D}}$ for $v \in H^{k+1}(\Omega)$ and also obtain the estimate (5.5) (using Clément's interpolation operator). Thus an a priori error estimate can be derived provided that the following approximation property of $R^{h}$ holds:

$$
\left\|R^{h}\left(\widetilde{\Pi}_{u}^{k, h}(v)\right)-\partial_{n} v\right\|_{0, \Gamma_{D}} \leq C h^{k-1 / 2}\|v\|_{k+1, \Omega}
$$

TheOREM 5.3. Let $\widetilde{V}^{h}$ and $\widetilde{W}^{h}$ be defined by (5.14) and (5.15), respectively. Let $(u, \lambda)$ be the solution to Problem (2.5) such that $u \in H^{k+1}(\Omega)$ and $\lambda \in H^{k-1 / 2}\left(\Gamma_{D}\right)$ for $k=\min \left\{k_{u}, k_{\lambda}+1\right\}$. Assume that (5.1) and (5.17) are satisfied. Then the following estimate holds:

$$
\text { | }\left\|\left(u-u^{h}, \lambda-\lambda^{h}\right)\right\| \mid \leq C h^{k}\|u\|_{k+1, \Omega},
$$

where $\left(u^{h}, \lambda^{h}\right)$ is the solution to Problem (5.2).

REMARK 3. Note that for $k_{\lambda} \geq 1$ the use of $\widetilde{W}^{h} \cap \mathcal{C}(\widetilde{\Omega})$ instead of $\widetilde{W}^{h}$ does not change the result. Note also that the definition of the norm $\left|\left\|\left(u-u^{h}, \lambda-\lambda^{h}\right)\right\|\right|$ involves a standard error estimate for $\left\|u-u^{h}\right\|_{V}$. However, it does not provide an estimate of $\left\|\lambda-\lambda^{h}\right\|_{-1 / 2, \Gamma_{D}}$ but the one of $h^{1 / 2}\left\|\lambda-\lambda^{h}\right\|_{0, \Gamma_{D}}$. An additional optimal estimate of $\left\|u-u^{h}\right\|_{0, \Gamma_{D}}$ is also available without supplementary regularity assumptions. This is due to the use of the Pitkäranta technique [21]. Error estimates with natural norms instead of mesh dependent norms are also possible for the stabilized problem (see [3]). 
5.1. Case $R^{h}\left(v^{h}\right)=\partial_{n} v^{h}$ and an additional condition on the mesh. A natural choice for the operator $R^{h}$ is of course

$$
R^{h}\left(v^{h}\right)=\partial_{n} v^{h} \text { on } \Gamma_{D}
$$

which corresponds to the original method of Barbosa and Hughes. In this case, unfortunately, the stability condition (5.1) is verified only under an additional regularity assumption on the intersection of the mesh with $\Omega$. We denote by $\hat{T}$ a reference element such that $T=\tau_{T}(\hat{T})$ for all $T \in \mathcal{T}^{h}$, where $\tau_{T}$ is a regular affine transformation in $\mathbb{R}^{d}$. The assumption on the mesh can be expressed as follows (see [21] for a similar one):

There exists a radius $\hat{\rho}>0$ independent of $h$ such that for any

$T \in \mathcal{T}^{h}, T \cap \Omega \neq \emptyset$ the reference element $\hat{T}$ contains a ball $B\left(\hat{y}_{T}, \hat{\rho}\right)$ which satisfies $B\left(\hat{y}_{T}, \hat{\rho}\right) \subset \tau_{T}^{-1}(T \cap \Omega)$

Under this assumption, inequality (5.1) is satisfied for $\widetilde{V}^{h}$ defined by (5.14) (see the proof in Appendix B). Moreover, the following lemma says that (5.17) is also satisfied.

LEMMA 5.4. Let $\widetilde{V}^{h}$ be defined by (5.14), $R^{h}\left(v^{h}\right)=\partial_{n} v^{h}$ on $\Gamma_{D}$ and assume that (5.18) is satisfied. Then (5.17) is satisfied as well.

Proof. Recall that $k=\min \left(k_{u}, k_{\lambda}+1\right)$. Using (5.16) and standard interpolation error estimates one has for any $v \in H^{k+1}(\Omega)$ :

$$
\begin{aligned}
\left\|R^{h}\left(\widetilde{\Pi}_{u}^{k, h}(v)\right)-\partial_{n} v\right\|_{0, \Gamma_{D}}^{2} \leq & \sum_{T \in \mathcal{T} h}\left\|\nabla \widetilde{\Pi}_{u}^{k, h}(v)-\nabla v\right\|_{0, \Gamma_{D}}^{2} \cap T \\
\leq & C \sum_{T \in \mathcal{T}^{h}}\left(h^{-1}\left\|\nabla \widetilde{\Pi}_{u}^{k, h}(v)-\nabla T_{u}^{k}(v)\right\|_{0, T}^{2}\right. \\
& \left.\quad+h\left\|\nabla \widetilde{\Pi}_{u}^{k, h}(v)-\nabla T_{u}^{k}(v)\right\|_{1, T}^{2}\right) \\
\leq & C \sum_{T \in \mathcal{T}^{h}}\left(h^{-1}\left(h^{k}\left\|T_{u}^{k}(v)\right\|_{k+1, T}\right)^{2}+h\left(h^{k-1}\left\|T_{u}^{k}(v)\right\|_{k+1, T}\right)^{2}\right) \\
\leq & C h^{2 k-1}\|v\|_{k+1, \Omega}^{2} .
\end{aligned}
$$

We can deduce that if $R^{h}\left(v^{h}\right)=\partial_{n} v^{h}$ on $\Gamma_{D}$, the estimate of Theorem 5.3 holds provided that (5.18) is satisfied. This assumption however restricts the use of our fictitious domain approach. Indeed if, for instance, one wants to approximate an evolving boundary, the intersection of elements with the real domain will be arbitrary. The aim of the next section is to introduce an operator $R^{h}$ with a reinforced stability enabling us to work with an arbitrary domain.

5.2. Operator $R^{h}$ with a reinforced stability. We give here an example how to construct an operator $R^{h}$ ensuring both the approximation property (5.17) as well as the stability property (5.1) for an arbitrary intersection of the mesh $\mathcal{T}^{h}$ with the domain $\Omega$. The proposed construction is only local and quite simple to implement.

Let $\hat{\rho}>0$ be an a priori given small radius $(\hat{\rho}<<1)$. For each element $T \in \mathcal{T}^{h}$ such that $T \cap \Omega \neq \emptyset$, we will designate by $T^{\prime}$ either the element $T$ itself if there is a ball $B\left(\hat{y}_{T}, \hat{\rho}\right) \subset \tau_{T}^{-1}(T \cap \Omega)$ (a "good" element) or any neighbor element possessing this property if $T$ itself does not satisfy it ( $T$ is a "bad" element). 
The proposed operator $R^{h}$ will simply be equal to $\partial_{n} \bar{v}_{T^{\prime}, T}^{h}$ where $\bar{v}_{T^{\prime}, T}^{h}$ is either $\left.v^{h}\right|_{T}$ if $T^{\prime}=T$ or the natural extension of $\left.v^{h}\right|_{T^{\prime}}$ onto $T$ if $T^{\prime} \neq T$. Of course, $\hat{\rho}>0$ has to be sufficiently small such that $T^{\prime}$ always exists, which is not a big constraint.

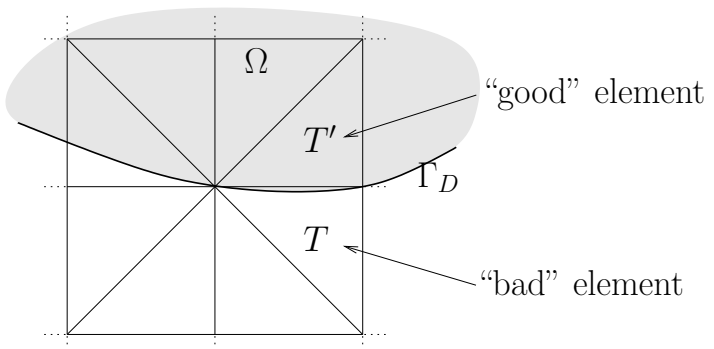

FIGURE 5.1. The choice of $T^{\prime}$ for an element $T$ having a small intersection with $\Omega$. In this case, it is more stable to evaluate the normal derivative from a natural extension of $v^{h}$ from $T^{\prime}$ on $T$ because smaller is the thickness of this intersection, poorer approximation of the normal derivative on $T \cap \partial \Omega$ is obtained using $v_{\mid T}^{h}$.

It is not difficult to see that the stability condition (5.1) is satisfied with such a choice of the operator $R^{h}$ (see Appendix C for the sketch of the proof). The following lemma establishes that (5.17) is also satisfied so that the estimate of Theorem 5.3 holds, again.

Lemma 5.5. Let $\widetilde{V}^{h}$ be defined by (5.14), and $R^{h}\left(v^{h}\right):=\partial_{n} \bar{v}_{T^{\prime}, T}^{h}$ on $\Gamma_{D}$. Then (5.17) is satisfied.

Proof. Suppose that $T$ is a "bad" element, i.e. $T \cap \Omega$ is "thin" and let $T$ ' be a "good" neighbor element as described above (see also Fig. 5.1). We prolong $T^{\prime}$ and construct the new element $T_{d}$ as shown in Fig. 5.2. The interpolation on $T_{d}$ is defined by the interpolation on $T^{\prime}$. More precisely:

$$
\text { let } v \in H_{\mathrm{loc}}^{k+1}\left(\mathbb{R}^{d}\right) \text { and } v_{T^{\prime}}:=v_{\left.\right|_{T^{\prime}}} \text {. }
$$

By $\Pi_{T^{\prime}} v_{T^{\prime}}$ we denote the $P_{k}$-Lagrange interpolant of $v_{T^{\prime}}$ constructed on $T^{\prime}$ (i.e. using degrees of freedom in $T^{\prime}$ ) but with the domain of definition being the whole $\mathbb{R}^{d}$. The interpolation of $v$ on $T_{d}$ is defined as:

$$
\Pi_{T_{d}} v:=\left.\Pi_{T^{\prime}} v_{T^{\prime}}\right|_{T_{d}}
$$

Classical arguments based on the fact that $v-\Pi_{T_{d}} v$ vanishes for all polynomials of degree less or equal $k$ lead to the following approximation property (see [6] for instance):

$$
\left\|v-\Pi_{T_{d}} v\right\|_{m, T_{d}} \leq C h_{T_{d}}^{k+1-m}\|v\|_{k+1, T_{d}} \quad\left(h_{T_{d}} \leq 2 h_{T^{\prime}}\right) .
$$




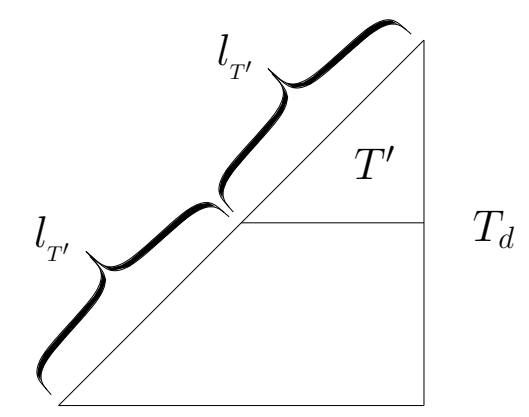

Figure 5.2. Prolongation of $T^{\prime}$.

Analogically to Lemma 8.1 (see Appendix A) it holds:

$$
\|v\|_{0, \Gamma_{D} \cap T_{d}}^{2} \leq C\left(h_{T_{d}}^{-1}\|v\|_{0, T_{d}}^{2}+h_{T_{d}}\|v\|_{1, T_{d}}^{2}\right) .
$$

To get (5.17) we proceed as in Lemma 5.4. Only we have to sort all elements into "good" and "bad" ones and to use either $\Pi_{T}$ or $\Pi_{T_{d}}$.

6. Some practical details for implementation. The implementation of the proposed method requires to overcome a certain number of difficulties. First of all, one has to select bases of the spaces $V^{h}$ and $W^{h}$ from the ones of $\widetilde{V}^{h}$ and $\widetilde{W}^{h}$. As far as $V^{h}$ is concerned, the task is rather easy because it suffices to select the basis functions among the ones of $\widetilde{V}^{h}$ which are not identically equal to zero in $\Omega$ (one can eventually remove those for which the intersection of their support with $\Omega$ is too small). It is a little more difficult to find a basis of the space $W^{h}$. Indeed, the traces on $\Gamma_{D}$ of basis functions of $\widetilde{W}^{h}$ may be linearly dependent, especially if $\Gamma_{D}$ is rectilinear. A possible way how to overcome this difficulty is to eliminate the redundant functions by analyzing the elementary mass matrices whose components are $\int_{\Gamma_{D} \cap T} \psi_{i} \psi_{j} d \Gamma$, where $\left\{\psi_{i}\right\}$ are the shape functions of $\widetilde{W}^{h}$.

Another difficulty concerns the numerical integration: one needs to build integration formulas on the intersection of elements with the domain $\Omega$ as well as on the intersection of elements with $\Gamma_{D}$. Our finite element library Getfem $++[22]$ uses splitting of elements into simplices in a conformal way with respect to $\partial \Omega$ and then it applies a standard integration formula on each sub-element. If $\partial \Omega$ is curved then some curved sub-elements can be used. One obtains an integration formula on $\Gamma_{D}$ by considering the faces of the sub-elements lying on $\Gamma_{D}$.

The natural extension of functions on "bad" elements which is needed to obtain the fully stabilized method described in Section 5.2 consists in seeking information in a "good" nearby element. This can be a handicap for certain finite element codes where calculations are done only elementwise. A possible remedy is to precompute a global discrete extension operator which gives the solution extended to "bad" elements from the original one. Then, the matrices involving $R^{h}\left(v_{h}\right)$ are obtained as a composition of classical matrices with this discrete extension operator.

The Xfem method is often associated with the use of some level-sets of functions defined on the mesh. This is particularly usefull when, for instance, one needs to represent an evolving interface. In our case such a level-set can be utilized to represent the boundary of $\Omega$. The implementation in Getfem ++ uses this strategy. Generally, 
this involves an additional approximation of $\Omega$. In our numerical tests presented in the next section, the level-set functions are piecewise second degree polynomials. In this case the level-set approach has no influence on the rate of convergence of the used finite element methods which are of the first and second order.

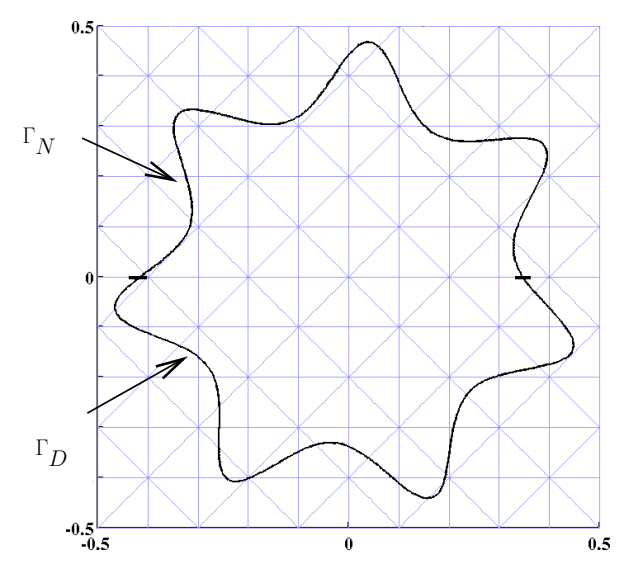

Figure 6.1. Test domain and a triangular structured mesh.

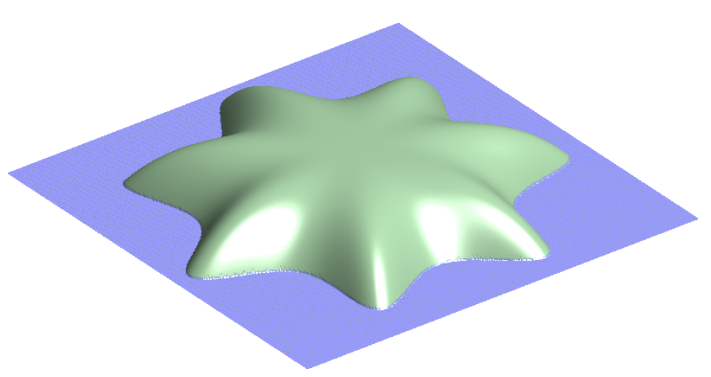

FIGURE 6.2. Exact solution.

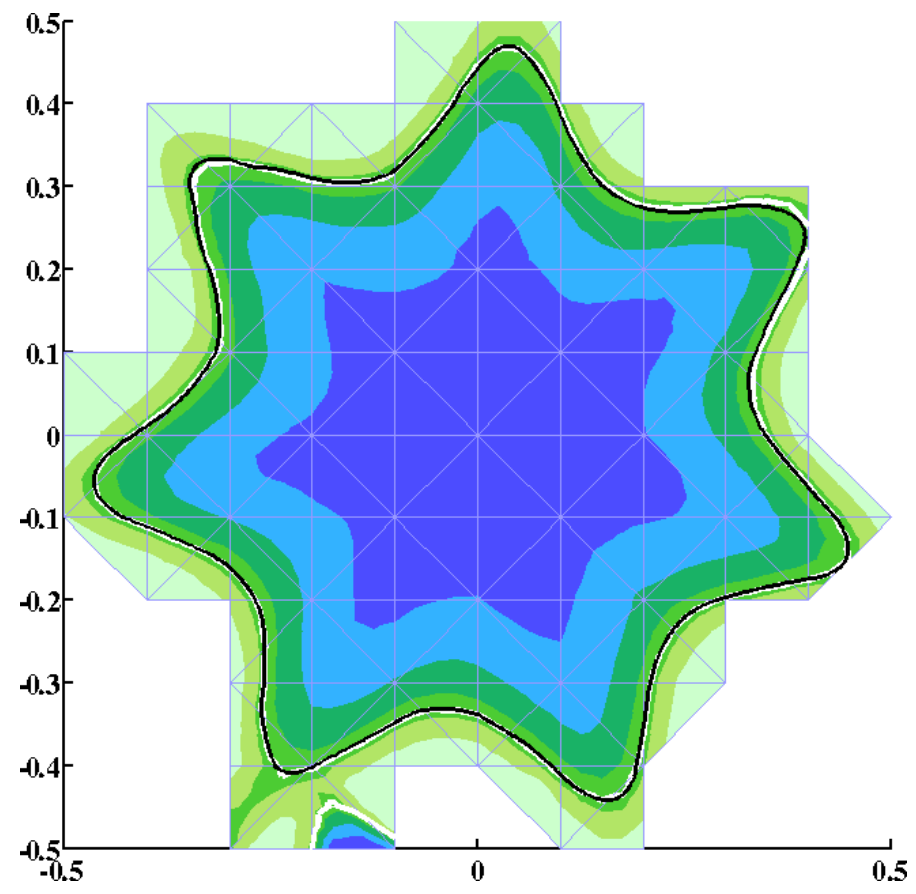

FIGURE 6.3. Approximated solution on a rough mesh with the $P_{2} / P_{1}$ method. Only the elements intersecting $\Omega$ are depicted. The black curve is the boundary of $\Omega$ and the white curve is the zero level-set of the approximated solution. 
7. Numerical experiments. In this section we present 2D-numerical tests. The fictitious domain is $\widetilde{\Omega}=]-1 / 2,1 / 2\left[{ }^{2}\right.$. The exact solution is $u(x)=R^{4}-|x|^{4}(5+$ $\left.3 \sin \left(7 \theta+\frac{7 \pi}{36}\right)\right) / 2$, where $R=0.47$ and $\theta(x)=\arctan \left(x_{2} / x_{1}\right)$. The real domain is $\Omega=\left\{x \in \mathbb{R}^{2}: u(x)<0\right\}$ and the Dirichlet and Neumann boundary conditions are defined on $\Gamma_{D}=\Gamma \cap\left\{x \in \mathbb{R}^{2}: x_{2}<0\right\}$ and $\Gamma_{N}=\Gamma \cap\left\{x \in \mathbb{R}^{2}: x_{2}>0\right\}$.

The domain $\Omega$ is represented in Fig. 6.1 with an example of a triangular structured mesh. The exact solution is shown in Fig. 6.2 while a computed solution on a rough mesh is depicted in Fig. 6.3.

7.1. without stabilization. First, we present numerical tests without any stabilization. We tested several choices of the finite element spaces $\widetilde{V}_{h}$ and $\widetilde{W}_{h}$.

In order to avoid the locking phenomena, the couple of selected finite element spaces should satisfy as much as possible a discrete mesh independent inf-sup condition since the stabilization is not used. For instance, it is known that the $P_{1} / P_{0}$ method for the discretization of $u, \lambda$, respectively, does not satisfy such a condition.

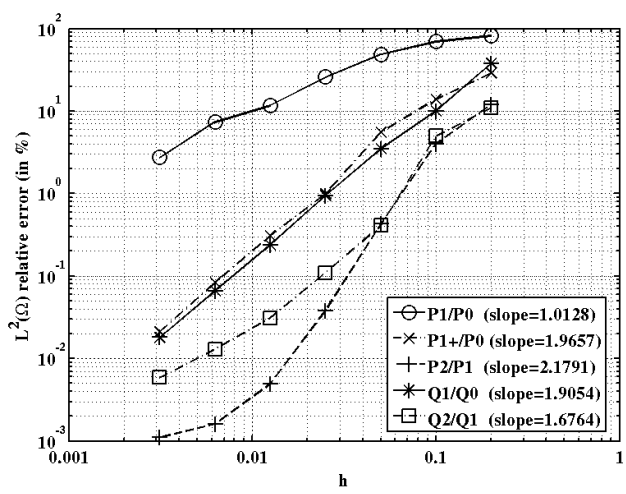

Rate of convergence $\left\|u-u^{h}\right\|_{0, \Omega}$.

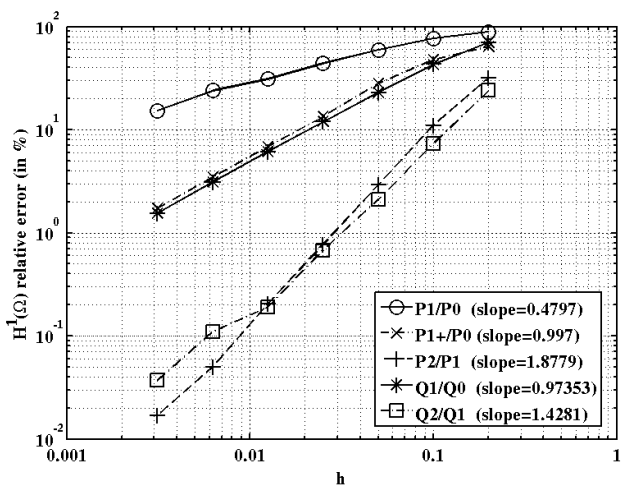

Rate of convergence $\left\|u-u^{h}\right\|_{1, \Omega}$.

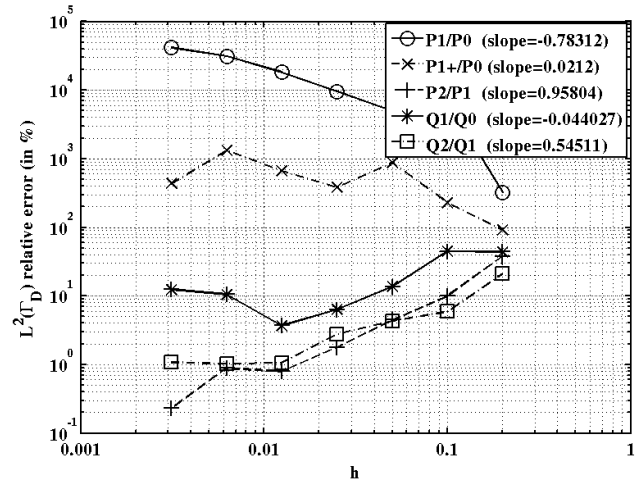

Rate of convergence $\left\|\lambda-\lambda^{h}\right\|_{0, \Gamma_{D}}$.

FIGURE 7.1. Rates of convergence for some couples of finite element spaces with no stabilization.

The linear system to be solved is of the form

$$
\left(\begin{array}{cc}
K & B^{T} \\
B & 0
\end{array}\right)\left(\begin{array}{l}
U \\
\Lambda
\end{array}\right)=\left(\begin{array}{l}
L \\
0
\end{array}\right),
$$


where $U$ and $\Lambda$ are the degrees of freedom of $u^{h}$ and $\lambda^{h}$, respectively, and the components of $K, B$ and $L$ are

$$
K_{i j}=\int_{\Omega} \nabla \varphi_{i} . \nabla \varphi_{j} d \Omega, \quad B_{i j}=\int_{\Gamma_{D}} \psi_{i} \varphi_{j} d \Gamma, \quad L_{i}=\int_{\Omega} f \varphi_{i} d \Omega+\int_{\Gamma_{N}} g \varphi_{i} d \Gamma,
$$

with $\left\{\varphi_{i}\right\},\left\{\psi_{j}\right\}$ being the selected basis functions of $\widetilde{V}_{h}, \widetilde{W}_{h}$, respectively. In our experiments, this system is solved using the library Superlu [9] (a direct LU solver for sparse matrices).

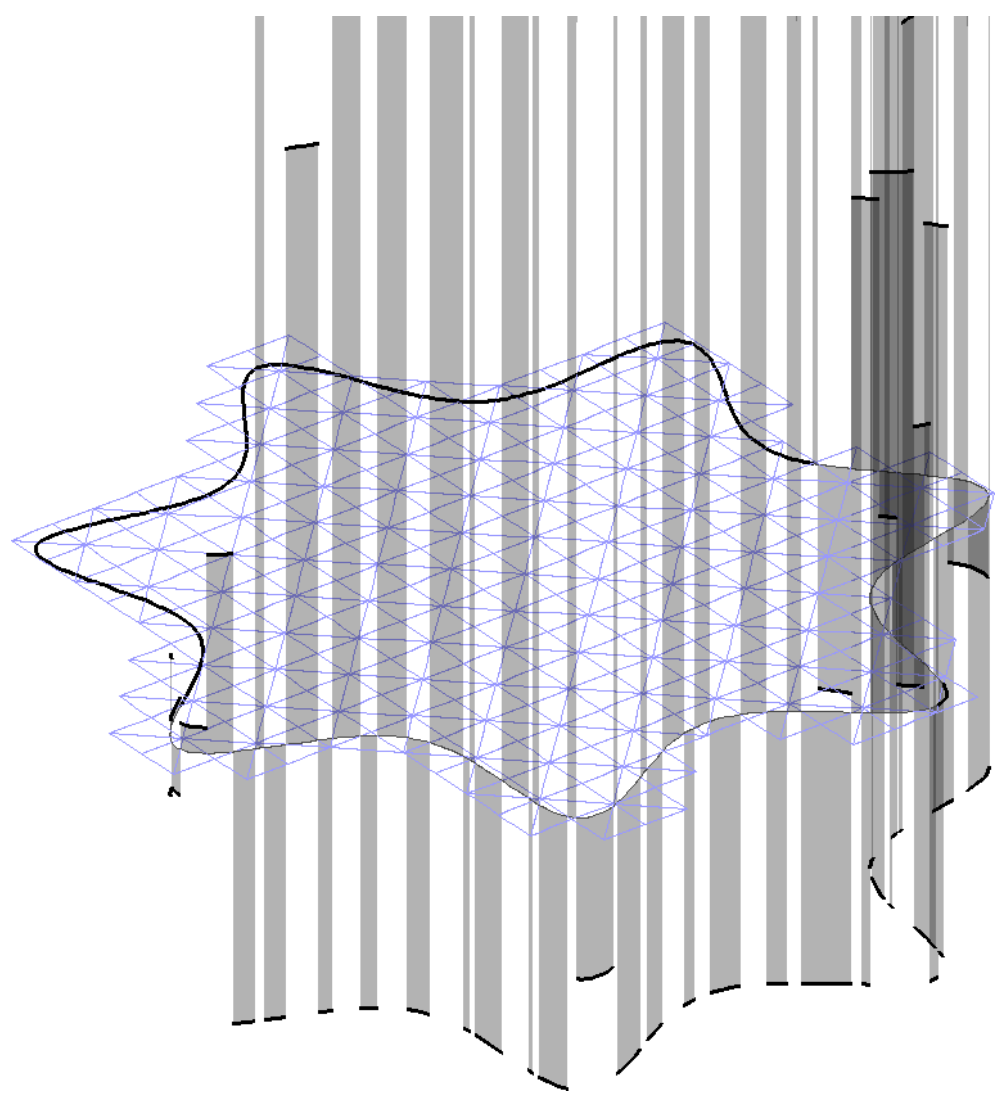

Figure 7.2. Multiplier on $\Gamma_{D}$ with no stabilization for the $P_{1} / P_{0}$ method $(h=0.05)$.

The couples of spaces tested are the following: $P_{1} / P_{0}, P_{1}+/ P_{0}$ (a standard continuous $P_{1}$ element for $u$ enriched by a cubic bubble function and a standard $P_{0}$ element for the multiplier), $Q_{1} / Q_{0}$ (standard continuous $Q_{1}$ and discontinuous $Q_{0}$ elements on quadrilaterals), $P_{2} / P_{1}, P_{2} / P_{0}$ and $Q_{2} / Q_{1}$.

Rates of convergence are presented in Fig. 7.1. One can see that in all experiments the rate of convergence in the $H^{1}(\Omega)$-norm is better than the theoretical one given by Proposition 3 except for the $P_{1} / P_{0}$ case which is a little bit slower than $h^{1 / 2}$. The choice $P_{1} / P_{0}$ suffers of course from the non-satisfaction of a mesh-independent inf-sup condition. It has to be stressed that in all the experiments without stabilization, and particularly for the $P_{1} / P_{0}$ case, a singular linear system can be obtained. However, in all examples, presented here, we selected some cases with a non-singular linear 
system. It is also seen that convergence of the multiplier is not generally obtained, especially for degree one methods. Fig. 7.2 illustrates a poor quality of the multiplier for the $P_{1} / P_{0}$ method. The $P_{2} / P_{1}$ method gives slightly better results (see Fig. 7.3 still with some oscillations in parts where the intersection of the element with the domain $\Omega$ is very small).

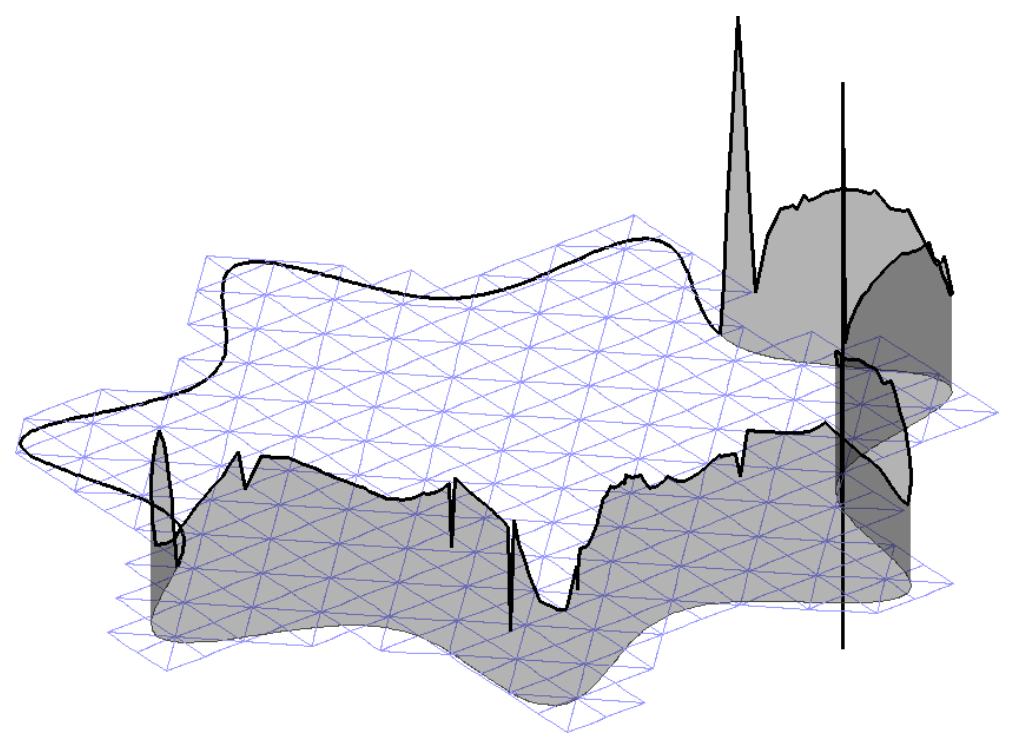

Figure 7.3. Multiplier on $\Gamma_{D}$ with no stabilization for the $P_{2} / P_{1}$ method $(h=0.05)$.

The test program can be downloaded on the Getfem++ web site [22]. It allows to test many other couples of elements and to treat also 3D-problems.

7.2. The stabilized method with $R^{h}\left(v^{h}\right)=\partial_{n} v^{h}$. The numerical experiments are now done using the standard Barbosa-Hughes stabilization technique (with $\gamma=$ 0.1 ). It has been proven in Section 5.1 that this method is optimal whenever the intersection of elements with the domain $\Omega$ is not too small. This is not easy to satisfy in computations. Of course, one way how to avoid small intersections would be to move a little bit some mesh nodes, at least when a structured mesh is not required. We did not test this possibility.

Unlike (7.1), the linear system to be solved is now of the form

$$
\left(\begin{array}{cc}
K_{\gamma} & B_{\gamma}^{T} \\
B_{\gamma} & -M_{\gamma}
\end{array}\right)\left(\begin{array}{c}
U \\
\Lambda
\end{array}\right)=\left(\begin{array}{c}
L \\
0
\end{array}\right)
$$

where the components of $K_{\gamma}, B_{\gamma}$ and $M_{\gamma}$ are

$$
\begin{gathered}
\left(K_{\gamma}\right)_{i j}=\int_{\Omega} \nabla \varphi_{i} . \nabla \varphi_{j} d \Omega-\gamma \int_{\Gamma_{D}} R^{h}\left(\varphi_{i}\right) R^{h}\left(\varphi_{j}\right) d \Gamma, \quad\left(B_{\gamma}\right)_{i j}=\int_{\Gamma_{D}} \psi_{i}\left(\varphi_{j}-\gamma R^{h}\left(\varphi_{j}\right)\right) d \Gamma, \\
\left(M_{\gamma}\right)_{i j}=\gamma \int_{\Gamma_{D}} \psi_{i} \psi_{j} d \Gamma,
\end{gathered}
$$

respectively. Note that $K_{\gamma}$ is invertible provided that $\gamma$ is sufficiently small. The whole matrix of the system is invertible as well whatever is $B_{\gamma}$. 


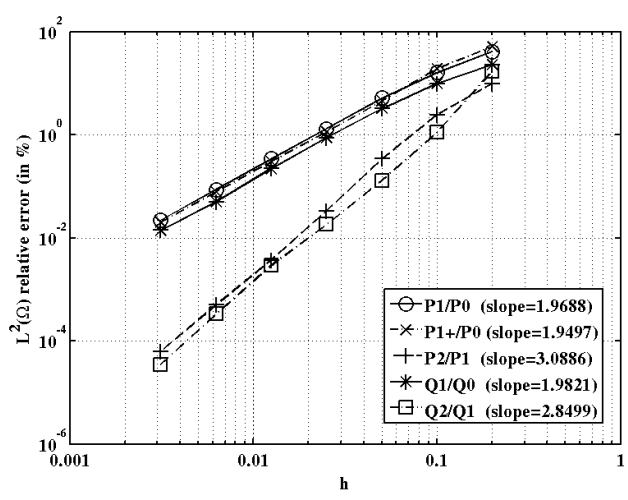

Rate of convergence $\left\|u-u^{h}\right\|_{0, \Omega}$.

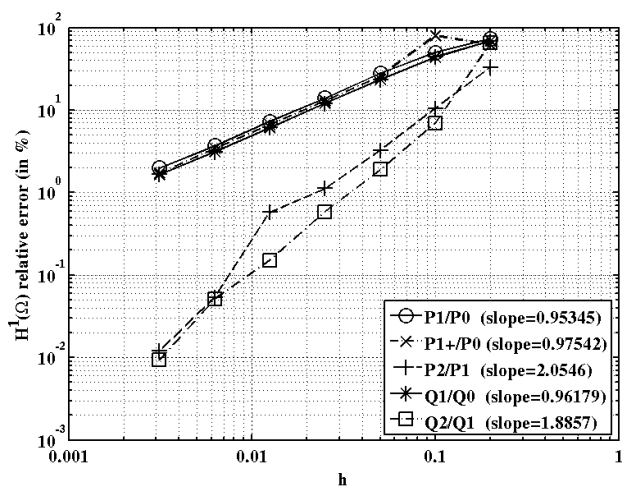

Rate of convergence $\left\|u-u^{h}\right\|_{1, \Omega}$.

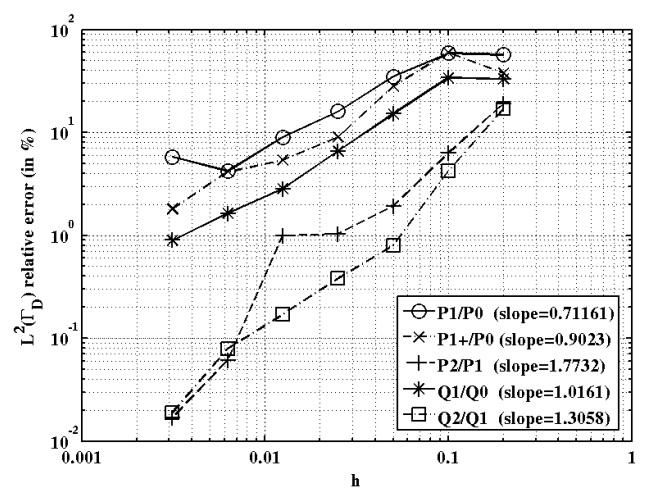

Rate of convergence $\left\|\lambda-\lambda^{h}\right\|_{0, \Gamma_{D}}$.

FIGURE 7.4. Rates of convergence for some couples of finite element spaces with the BarbosaHughes stabilization.

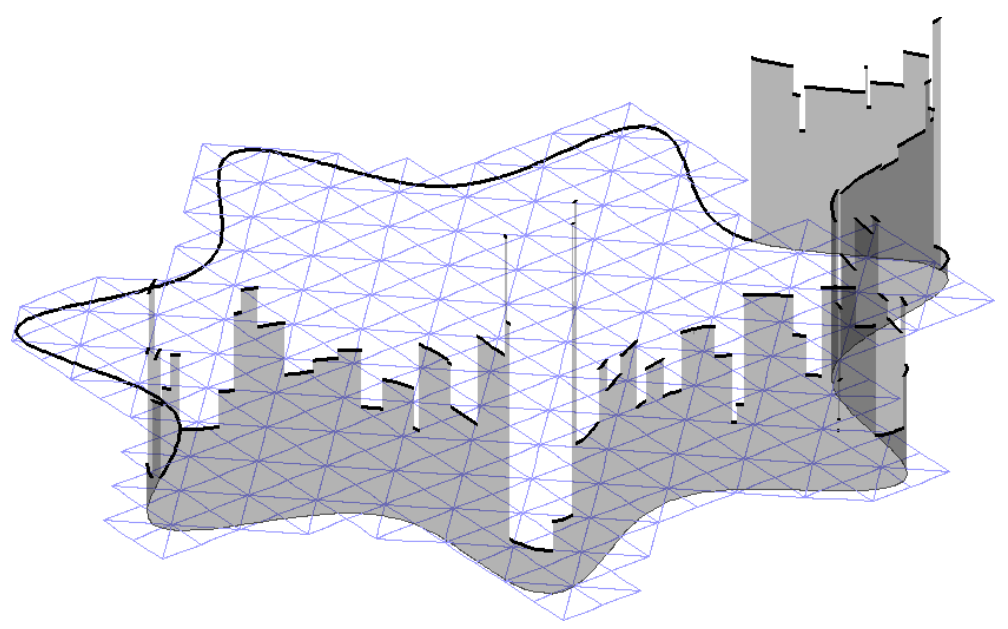

Figure 7.5. Multiplier on $\Gamma_{D}$ with the Barbosa-Hughes stabilization for the $P_{1} / P_{0}$ method $(h=0.05)$. 
Rates of convergence are presented in Fig. 7.4 for the same couples of elements as in the previous section. The stabilization significantly improves the convergence of the $P_{1} / P_{0}$ choice (the stabilization with bubble functions is no longer necessary) and the convergence of quadratic elements. Moreover, the linear system is guaranteed to be invertible. Fig. 7.5 shows that also the approximation of the multiplier is considerably improved. The convergence rate is improved by the stabilization, but some problems remain with too small intersections of elements with $\Omega$ even for degree two methods (see Fig. 7.6).

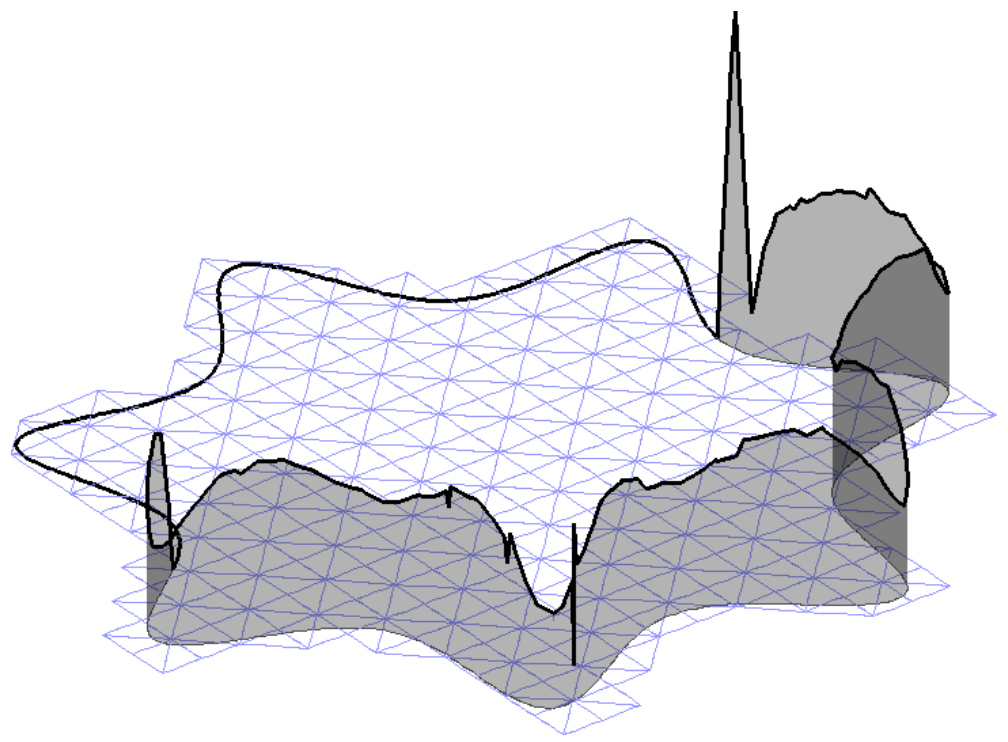

FIGURE 7.6. Multiplier on $\Gamma_{D}$ with the Barbosa-Hughes stabilization for the $P_{2} / P_{1}$ method $(h=0.05)$.

7.3. The fully stabilized method. We now consider the fully stabilized method described in Section 5.2. An element $T$ is considered to be "bad" when $|T \cap \Omega|$ is less than one percent of $|T|$. We see that the multipliers behave in a more regular way than before. The convergence curves given in Fig. 7.7 are rather the same than with the standard Barbosa-Hughes stabilization used in the previous section. The difference lies only on the elements having a too small intersection with the domain. 


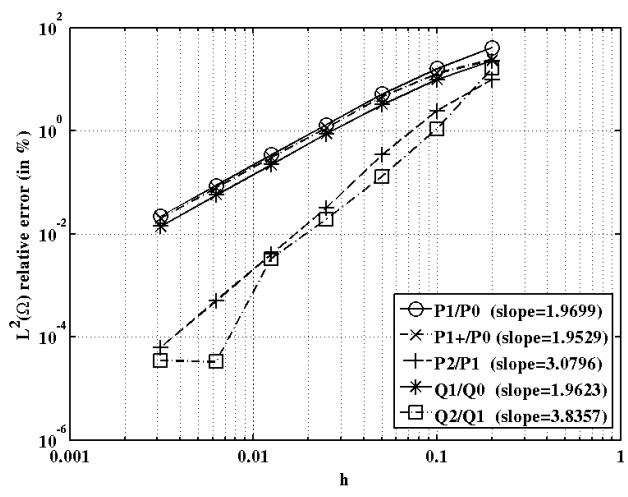

Rate of convergence $\left\|u-u^{h}\right\|_{0, \Omega}$.

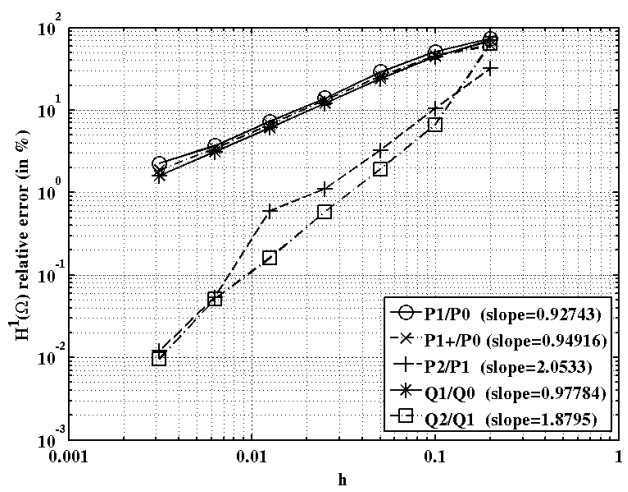

Rate of convergence $\left\|u-u^{h}\right\|_{1, \Omega}$.

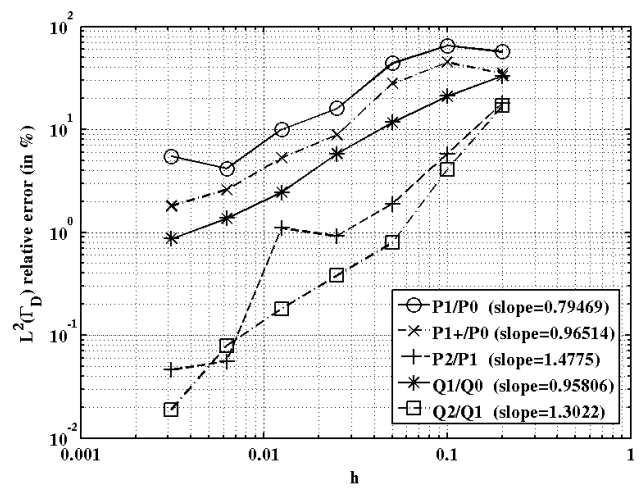

Rate of convergence $\left\|\lambda-\lambda^{h}\right\|_{0, \Gamma_{D}}$.

Figure 7.7. Rates of convergence for some couples of finite element spaces with the fully stabilized method.

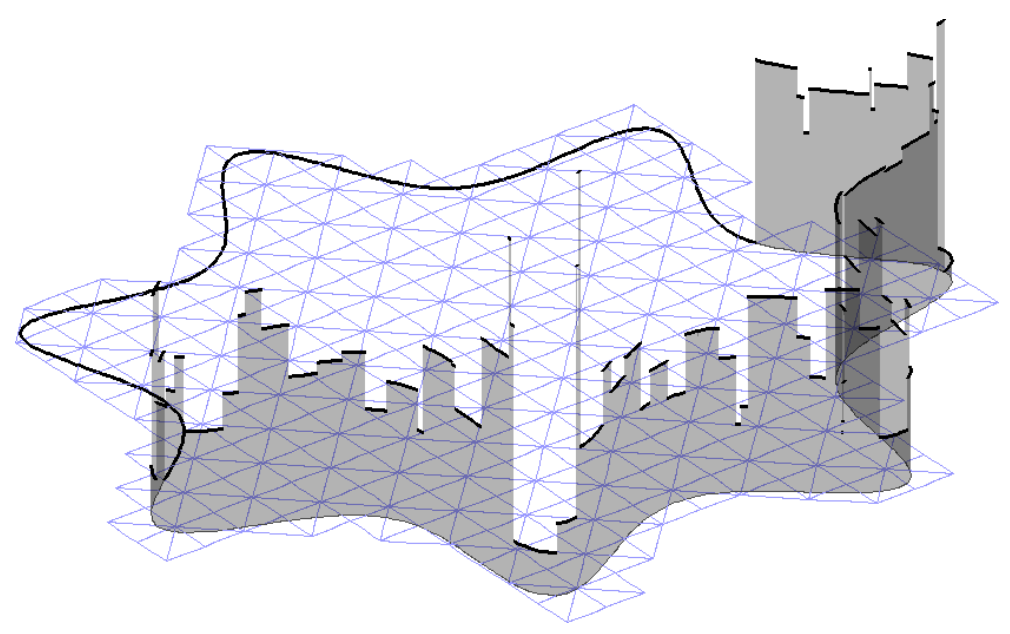

FIGURE 7.8. Multiplier on $\Gamma_{D}$ with the fully stabilized method for the $P_{1} / P_{0}$ method $(h=0.05)$. 


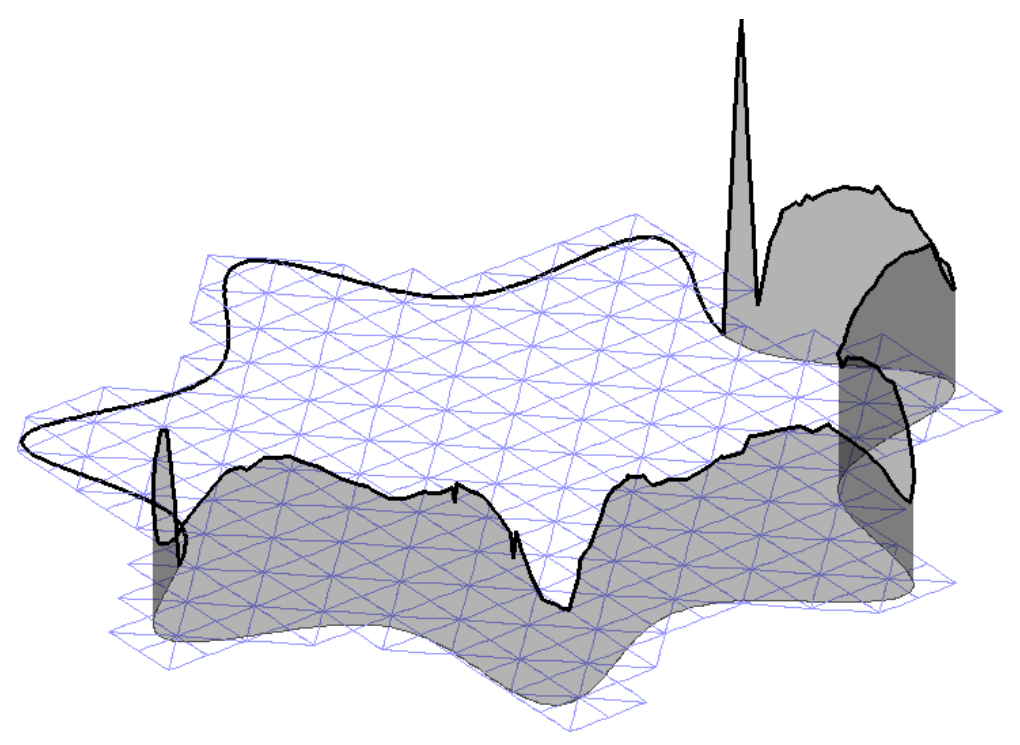

FIGURE 7.9. Multiplier on $\Gamma_{D}$ with the fully stabilized method for the $P_{2} / P_{1}$ method $(h=0.05)$.

8. Concluding remarks. In this paper, we combined the Xfem approach together with the Barbosa-Hughes stabilized formulation to get a new fictitious domain method. This method is quite simple to implement since all the variables (multipliers and primal variables) are defined on a single mesh independent of the computational domain. Moreover, it potentially allows to treat complex boundary conditions (such as contact and friction).

The fully stabilized method introduced in Section 5.2 leads to a robust method in the sense that it converges whatever is the intersection of the domain with the mesh. This is not the case if the Barbosa-Hughes stabilization technique is used alone, for which the quality of the approximation of the multiplier cannot be guaranteed on the elements having a too small intersection with the domain. Note that in [21] a similar approach is presented. However, the error estimate is given under the assumption (5.18) and the definition of multipliers requires the construction of a quasi-uniform family of meshes on the boundary.

Acknowledgments. The first author acknowledges MSM0021620839 of the Czech Ministry of Education and IAA100750802 of GAAV CR

This work is supported by "l'Agence Nationale de la Recherche", project ANR-05JCJC-0182-01.

Many thanks to Julien Pommier for his participation to obtain nice numerical experiments.

Appendix A. In this appendix we prove the trace inequality (5.16). For a proof in a more classical framework see for instance [12]. The proof is done by scaling with respect to a reference element $\hat{T}$.

We recall that for all $T \in \mathcal{T}^{h}$ one has $T=\tau_{T}(\hat{T})$, where $\tau_{T}$ is an affine and invertible mapping in $\mathbb{R}^{d}$. We make the following hypotheses: 
a) $\Gamma_{D}$ is a Lipschitz-continuous boundary.

b) there exists a constant $C_{2}>0$ independent of $h$ and $T \in \mathcal{T}^{h}$ such that $\left\|\nabla \tau_{T}\right\|_{\infty, T} \leq C_{2} h_{T}$ and $\left\|\nabla \tau_{\hat{T}}^{-1}\right\|_{\infty, T} \leq C_{2} h_{T}^{-1}$.

These two hypotheses are satisfied for regular families of meshes provided that $\Gamma_{D}$ is Lipschitz-continuous.

Lemma 8.1. Let a) and b) be satisfied. Then there exists a constant $C>0$ independent of $h$ and $T \in \mathcal{T}^{h}$ such that

$$
\|v\|_{0, \Gamma_{D} \cap T}^{2} \leq C\left(h_{T}^{-1}\|v\|_{0, T}^{2}+h_{T}\|v\|_{1, T}^{2}\right), \quad \forall v \in H^{1}(T) .
$$

Proof. Since $\mathcal{C}^{\infty}(T)$ is dense in $H^{1}(T)$ one can confine to functions $v \in \mathcal{C}^{\infty}(T)$. Denoting $\hat{\Gamma}_{D}=\tau_{T}^{-1}\left(\Gamma_{D} \cap T\right)$ and $\hat{\mathbf{n}}$ a unit normal vector to $\hat{\Gamma}_{D}$, one has:

$$
\int_{\Gamma_{D} \cap T} v^{2} d \Gamma=\int_{\hat{\Gamma}_{D}} \hat{v}^{2}\left|\operatorname{det}\left(\nabla \tau_{T}\right)\right|\left\|\nabla \tau_{T}^{-1} \hat{\mathbf{n}}\right\| d \hat{\Gamma} \leq C h_{T}^{d-1} \int_{\hat{\Gamma}_{D}} \hat{v}^{2} d \hat{\Gamma}
$$

where $\hat{v}=v \circ \tau_{T}$. Let us prove now that the following trace inequality

$$
\int_{\hat{\Gamma}_{D}} \hat{v}^{2} d \hat{\Gamma} \leq C_{3}\|\hat{v}\|_{1, \hat{T}}^{2} \quad \forall \hat{v} \in \mathcal{C}^{\infty}(\hat{T}),
$$

is such that the constant $C_{3}>0$ does not depend on the position of $\hat{\Gamma}_{D}$ inside of $\hat{T}$. This has been proved for a straight intersection in [11]. Let us consider the case $\hat{\Gamma}_{D}$ curved. For a sufficiently small mesh parameter $h$ the curve $\hat{\Gamma}_{D}$ is a graph of a function over a segment $\hat{l}$ contained in $\hat{T}$. Without loss of generality we may assume that $\hat{l}$ coincides with the $\hat{x}$-axis (after appropriate shift and rotation of $\hat{T}$ ). Then $\Gamma_{D}$ can be parametrized by the mean of a function $(\hat{x}, a(\hat{x})), \hat{x} \in \hat{l}$ and one has

$$
\hat{v}(\hat{x}, a(\hat{x}))=\hat{v}(\hat{x}, 0)+\int_{0}^{a(\hat{x})} \frac{\partial}{\partial_{y}} \hat{v}(\hat{x}, \tau) d \tau .
$$

thus

$$
\hat{v}^{2}(\hat{x}, a(\hat{x})) \leq C\left(\hat{v}^{2}(\hat{x}, 0)+\int_{0}^{a(\hat{x})}\left(\frac{\partial}{\partial_{y}} \hat{v}(\hat{x}, \tau)\right)^{2} d \tau\right)
$$

where $C>0$ is an absolute constante. Integrating over $\hat{l}$ we obtain

$$
\begin{aligned}
\int_{\hat{l}} \hat{v}^{2}(\hat{x}, a(\hat{x})) d \hat{x} & \leq C\left(\int_{\hat{l}} \hat{v}^{2}(\hat{x}, 0) d \hat{x}+\int_{\hat{T}}\left(\frac{\partial}{\partial y} \hat{v}(\hat{x}, \tau)\right)^{2} d \tau d \hat{x}\right) \\
& \leq C\|v\|_{1, \hat{T}}
\end{aligned}
$$

using the result for the straigh segment $\hat{l}$. Now, we can conclude by the fact that

$$
\begin{aligned}
\int_{\hat{\Gamma}_{D}} \hat{v}^{2} d \hat{\Gamma} & =\int_{\hat{l}} \hat{v}^{2}(\hat{x}, a(\hat{x})) \sqrt{1+\left(a^{\prime}(\hat{x})\right)^{2}} d \hat{x} \\
& \leq \max _{\hat{l}} \sqrt{1+\left(a^{\prime}(\hat{x})\right)^{2}} \int_{\hat{l}} \hat{v}^{2}(\hat{x}, a(\hat{x})) d \hat{x}
\end{aligned}
$$

since $\Gamma_{D}$ is assumed to be Lipschitz-continuous. 
Using now (8.1) and $\|\nabla \hat{v}\|_{\infty, \hat{T}} \leq C h_{T}\|\nabla v\|_{\infty, T}$ we can establish the estimate of the lemma:

$$
\begin{aligned}
\|v\|_{0, \Gamma_{D} \cap T}^{2} & \leq C h_{T}^{d-1} \int_{\hat{T}}\left(\hat{v}^{2}+|\hat{\nabla} \hat{v}|^{2}\right) d \hat{x} \\
& \leq C h_{T}^{-1} \int_{\hat{T}}\left(\hat{v}^{2}+|\hat{\nabla} \hat{v}|^{2}\right)\left|\operatorname{det}\left(\nabla \tau_{T}\right)\right| d \hat{x} \\
& \leq C h_{T}^{-1} \int_{T} v^{2} d x+C h_{T} \int_{T}|\nabla v|^{2} d x .
\end{aligned}
$$

Appendix B. We prove the discrete trace inequality (5.1) when $R^{h}\left(v^{h}\right)=\partial_{n} v^{h}$ provided that (5.18) is satisfied under the same hypotheses on the family of meshes and on $\Gamma_{D}$ as in Appendix A. First we prove the following auxiliary result.

Lemma 8.2. Let $v^{h}$ be defined on $T \in \mathcal{T}^{h}$ by $v^{h}(x):=\hat{v}\left(\tau_{T}^{-1}(x)\right)$ with $\hat{v} \in P_{k}\left(\mathbb{R}^{d}\right)$ and suppose that (5.18) is satisfied. Then there exists a constant $C>0$ independent of $h, T$ and $\hat{v}$ such that

$$
\int_{\Gamma_{D} \cap T}\left(v^{h}\right)^{2} d \Gamma \leq C h_{T}^{-1} \int_{\Omega \cap T}\left(v^{h}\right)^{2} d x
$$

Proof. Because of the equivalence of norms on $P_{k}\left(\mathbb{R}^{d}\right)$, one has

$$
\begin{aligned}
\|\hat{v}\|_{\infty, \hat{T}}^{2} & \leq\|\hat{v}\|_{\infty, B\left(\hat{y}_{T}, 2\right)}^{2}=\left\|\hat{v} \circ t_{\left(-\hat{y}_{T}\right)}\right\|_{\infty, B(0,2)}^{2} \\
& \leq C\left\|\hat{v} \circ t_{\left(-\hat{y}_{T}\right)}\right\|_{0, B(0, \hat{\rho})}^{2}=C\|\hat{v}\|_{0, B\left(\hat{y}_{T}, \hat{\rho}\right)}^{2} \leq C \int_{\tau_{T}^{-1}(T \cap \Omega)} \hat{v}^{2} d \hat{x},
\end{aligned}
$$

where $t_{\left(-\hat{y}_{T}\right)}$ is the translation defined by $t_{\left(-\hat{y}_{T}\right)}(x)=x-\hat{y}_{T}$. Thus, still with notations of Appendix A:

$$
\begin{aligned}
\int_{\Gamma_{D} \cap T}\left(v^{h}\right)^{2} d \Gamma & =\int_{\hat{\Gamma}_{D}} \hat{v}^{2}\left|\operatorname{det}\left(\nabla \tau_{T}\right)\right|\left\|\nabla \tau_{T}^{-1} \hat{\mathbf{n}}\right\| d \hat{\Gamma} \leq C h_{T}^{d-1}\|\hat{v}\|_{\infty, \hat{T}}^{2}\left|\hat{\Gamma}_{D}\right| \\
& \leq C \frac{h_{T}^{d-1}}{h_{T}^{d}} \int_{\tau_{T}^{-1}(T \cap \Omega)} \hat{v}^{2}\left|\operatorname{det}\left(\nabla \tau_{T}\right)\right| d \hat{x}=C h_{T}^{-1} \int_{T \cap \Omega}\left(v^{h}\right)^{2} d x .
\end{aligned}
$$

Now, summing up the previous estimate over elements of $\mathcal{T}^{h}$ one obtains the following result.

Lemma 8.3. Let $v^{h}$ be defined on $\Omega$ by $v^{h}(x)_{T}=\hat{v}_{T}\left(\tau_{T}^{-1}(x)\right), \hat{v}_{T} \in P_{k}\left(\mathbb{R}^{d}\right)$, $T \in \mathcal{T}^{h}$ and suppose that (5.18) is satisfied. Then the following estimate holds with a constant $C>0$ independent of $h$ and $v^{h}$ :

$$
h \int_{\Gamma_{D}}\left(v^{h}\right)^{2} d \Gamma \leq C \int_{\Omega}\left(v^{h}\right)^{2} d x .
$$

The discrete trace inequality (5.1) can be now easily deduced since $\left\|\partial_{n} v^{h}\right\|_{0, \Gamma_{D}} \leq$ $\left\|\nabla v^{h}\right\|_{0, \Gamma_{D}}$ and for a quasi-uniform family of meshes the previous lemma can be applied to $\nabla v^{h}$ componentwise. 
Appendix C. We now adapt the proof of Appendix B to the operator $R^{h}\left(v^{h}\right)$ defined in Section 5.2. The difference comes from those elements $T \in \mathcal{T}^{h}$ having a too small intersection with $\Omega$ ("bad" elements) and for which a neighbor element $T$ " has been selected to make a natural extension of functions. For such an element, the proof of Appendix B has to be modified because we evaluate the polynomial on a larger zone than $\hat{T}=\tau_{T^{\prime}}^{-1}\left(T^{\prime}\right)$ namely on $\hat{T}_{T, T^{\prime}}=\tau_{T^{\prime}}^{-1}\left(T^{\prime} \cup(T \cap \Omega)\right)$. With the quasi-uniform assumption for the meshes, it is readily seen that this zone is included in $\hat{T}_{\rho_{R}}=\left\{x \in \mathbb{R}^{d}: \operatorname{dist}(x, \hat{T}) \leq \rho_{R}\right\}$ for some $\rho_{R}>0$ independent of $h, T$ and $T^{\prime}$. Lemma 8.2 can be easily adapted remarking that there exists a constant $C>0$ independent of $h$ such that

$$
\|\hat{v}\|_{\infty, \hat{T}_{\rho_{R}}} \leq C\|\hat{v}\|_{\infty, \hat{T}} \quad \forall \hat{v} \in P_{k}\left(\mathbb{R}^{d}\right),
$$

using again that all norms are equivalent in $P_{k}\left(\mathbb{R}^{d}\right)$. From this the estimate

$$
\int_{\Gamma_{D} \cap T}\left(v^{h}\right)^{2} d \Gamma \leq C h_{T}^{-1} \int_{\Omega \cap T^{\prime}}\left(v^{h}\right)^{2} d x
$$

where $v^{h}(x):=\hat{v}\left(\tau_{T^{\prime}}^{-1}(x)\right), x \in \mathbb{R}^{d}$ follows. Thus (5.1) can be established remarking that the element $T^{T^{\prime}}$ can be selected as a neighbor element only a finite number times independently of $h$ still due to the quasi-uniform property of the meshes.

\section{REFERENCES}

[1] R.A. Adams, Sobolev spaces, Academic Press, 1975.

[2] H. J.C. Barbosa And T.J.R. Hughes. The finite element method with Lagrange multipliers on the boundary: circumventing the Babuška-Brezzi condition, Comput. Meth. Appl. Mech. Engrg., 85 (1991), 109-128.

[3] H. J.C. Barbosa And T.J.R. Hughes. Boundary Lagrange multipliers in finite element methods: error analysis in natural norms, Numer. Math., 62 (1992), 1-15.

[4] P. Hansbo, C. Lovadina, I. Perugia and G. Sangalli, A lagrange multiplier method for the finite element solution of elliptic interface problems using nonmatching meshes, Numer. Math., 100 (2005), pp. 91-115.

[5] E.Chahine, P. Laborde, Y. Renard. Crack-Tip enrichment in the Xfem method using a cut-off function. Submitted.

[6] P.G. Ciarlet, The finite element method for elliptic problems, Studies in Mathematics and its Applications No 4, North Holland, 1978.

[7] P.G. Ciarlet, The finite element method for elliptic problems, in Handbook of Numerical Analysis, Volume II, Part 1, eds. P.G. Ciarlet and J.L. Lions, North Holland, pp. 17-352, 1991.

[8] P. ClÉment, Approximation by finite elements functions using local regularization, RAIRO, Anal. Numer., 9 (1975), pp 77-84.

[9] J.W. Demmel, J.R. Gilbert, X.S. Li. a general purpose library for the direct solution of large, sparse, nonsymmetric systems, http://crd.lbl.gov/ xiaoye/SuperLU/.

[10] A. Ern, J.-L. Guermond. Theory and Practice of Finite Elements, Applied Mathematical Sciences, vol. 159, Springer 2004.

[11] V. Girault, R. Glowinski. Error analysis of a fictitious domain method applied to a Dirichlet problem, Japan J. Indust.Appl. Math. 12 (1995), pp. 487-514.

[12] P. GRISvARD. Elliptic problems in nonsmooth domains. Monographs and Studies in Mathematics. Pitman (Advanced Publishing Program), Boston, MA, 1985.

[13] J. Haslinger, A. Klarbring. Fictitious domain/mixed finite element approach for a class of optimal shape design problems. Math. Modelling and Numerical Analysis (M2AN), 29:4 (1995), pp. 435-450.

[14] P. Hild, Y. Renard. A stabilized Lagrange multiplier method for the finite element approximation of contact problems in elastostatics, submitted

[15] T. Hughes And L.P. FRAnCA, A new finite element formulation for computational fluid dynamics. VII. The Stokes problem with various well-posed boundary conditions: symmetric 
formulations that converge for all velocity/pressure spaces, Comput. Methods Appl. Mech. Engrg., 65 (1987), 85-96.

[16] P. Laborde, J. Pommier, Y. Renard, M. Salaün. High order extended finite element method for cracked domains. Int. J. Numer. Meth. Engng., 64 (2005), 354-381.

[17] N. Mö̈s, E. BÉchet, And M. Tourbier. Imposing Dirichlet boundary conditions in the eXtended Finite Element Method. Int. J. Numer. Meth. Engng., 67, 12 (2006), 354-381.

[18] N. MoËs, J. Dolbow, AND T. Belytschko. A finite element method for crack growth without remeshing. Int. J. Numer. Meth. Engng., 46 (1999), 131-150.

[19] N. MoËs, A. Gravouil and T. BelytschKo. Non-planar 3D crack growth by the extended finite element and level sets, Part I: Mechanical model. Int. J. Numer. Meth. Engng., 53, 11 (2002), 2549-2568.

[20] J. Nitsche, Über ein Variationsprinzip zur Lösung von Dirichlet-Problemen bei Verwendung von Teilräumen, die keinen Randbedingungen unterworfen sind, Abh. Math. Univ. Hamburg, 36 (1971), 9-15.

[21] J. Pitkäranta, Local stability conditions for the Babuška method of Lagrange multipliers, Mathematics of Computation, vol. 35 (1980), 152, 1113-1129.

[22] Y. Renard, J. Pommier Getfem++. An open source generic $C++$ library for finite element methods, http://home.gna.org/getfem/

[23] F.L. Stazi, E. Budyn, J. Chessa, and T. Belytschko. An extended finite element method with higher-order elements for curved cracks. Computational Mechanics, 31 (2003), 38-48.

[24] R. StenberG, On some techniques for approximating boundary conditions in the finite element method, J. Comput. Appl. Math., 63 (1995), 139-148.

[25] M. Stolarska, D.L. Chopp, N. Mö̈s, T. Belytschko. Modelling crack growth by level sets in the extended finite element method. Int. J. Numer. Meth. Engng., 51 (2001), 943-960.

[26] G. Strang, G. J. Fix. An Analysis of the Finite Element Method. Prentice-Hall, Englewood Cliffs, 1973.

[27] N. Sukumar, D. L. Chopp, N. MoËs, T. Belytschko. Modeling holes and inclusions by level sets in the extended finite element method. Comput. Meth. Appl. Mech. Eng., 90, 46 (2001), $6183-6200$.

[28] N. Sukumar, N. Moës, B. Moran and T. Belytschko. Extended finite element method for three dimensional crack modelling. Int. J. Numer. Meth. Engng., 48 (2000), 1549-1570. 